\title{
Instantons on Calabi-Yau and hyper-Kähler cones
}

\author{
Jakob C. Geipel ${ }^{a}$ and Marcus Sperling ${ }^{b}$ \\ ${ }^{a}$ Institut für Theoretische Physik, Leibniz Universität Hannover, \\ Appelstraße 2, 30167 Hannover, Germany \\ ${ }^{b}$ Fakultät für Physik, Universität Wien, \\ Boltzmanngasse 5, 1090 Wien, Austria \\ E-mail: jakob.geipel@itp.uni-hannover.de, \\ marcus.sperling@univie.ac.at
}

AbstRACT: The instanton equations on vector bundles over Calabi-Yau and hyper-Kähler cones can be reduced to matrix equations resembling Nahm's equations. We complement the discussion of Hermitian Yang-Mills (HYM) equations on Calabi-Yau cones, based on regular semi-simple elements, by a new set of (singular) boundary conditions which have a known instanton solution in one direction. This approach extends the classic results of Kronheimer by probing a relation between generalised Nahm's equations and nilpotent pairs/tuples. Moreover, we consider quaternionic instantons on hyper-Kähler cones over generic 3-Sasakian manifolds and study the HYM moduli spaces arising in this set-up, using the fact that their analysis can be traced back to the intersection of three Hermitian Yang-Mills conditions.

Keywords: Solitons Monopoles and Instantons, Differential and Algebraic Geometry, Gauge Symmetry

ArXiv EPRINT: 1709.01944 


\section{Contents}

1 Introduction 1

2 Geometry 3

3 Comments on moduli space of instantons 5

3.1 Space of connections over hyper-Kähler spaces 5

3.2 Equivalence of $\mathrm{Sp}(m)$-instantons and intersections of HYM instantons $\quad 7$

3.3 Quaternionic instantons 8

3.4 Ansatz for equivariant instantons 8

4 Instantons on Calabi-Yau cones $\quad 10$

$\begin{array}{lll}4.1 \text { Set-up } & 10\end{array}$

$\begin{array}{lll}4.2 & \text { Relation to coadjoint orbits } & 12\end{array}$

$\begin{array}{ll}4.3 \text { Singular boundary conditions } & 13\end{array}$

$\begin{array}{ll}4.4 \text { Nilpotent pairs and generalised Nahm's equations } & 15\end{array}$

5 Instantons on hyper-Kähler cones $\quad \mathbf{1 6}$

$\begin{array}{ll}5.1 \text { Explicit form of hyper-Kähler instanton equations } & 16\end{array}$

$\begin{array}{ll}5.2 & \text { Single HYM moduli space } \\ 5.3 & \text { Space of equivartant }\end{array}$

$\begin{array}{lll}5.3 & \text { Space of equivariant connections } & 23\end{array}$

$\begin{array}{lll}6 & \text { Summary and conclusions } & 24\end{array}$

$\begin{array}{ll}\text { A Details on non-regular boundary conditions } & 25\end{array}$

\section{Introduction}

Instantons and (hyper-)Kähler geometry are both interesting subjects for physicists as well as mathematicians, and important results in mathematical physics have been derived by studying the structure of moduli spaces of certain gauge connections.

The geometry of hyper-Kähler manifolds is in itself very restrictive and there is to this day no explicit compact hyper-Kähler metric known. Nonetheless, the classification of compact hyper-Kähler spaces is understood [1] and yields four classes: two series of $\mathrm{K} 3^{n}$ and generalised Kummer varieties, as well as two exceptional examples by O'Grady. In contrast, many examples of (non-compact) hyper-Kähler spaces arise as moduli spaces of gauge theory problems: moduli spaces of instantons, monopoles, or Hitchin equations, to name a few. As it turns out [2], hyper-Kähler geometry is intimately related to supersymmetry. Various moduli spaces of supersymmetric vacua are hyper-Kähler: including manifestations of the so-called hyper-Kähler quotient and of hyper-Kähler cones [3, 4]. 
Instantons on 4-manifolds, meaning (anti-)self-dual connections, led to an improved understanding via the concept of Donaldson invariants [5]. On the other hand, 4-dimensional Euclidean instantons are vital for non-perturbative effects in quantum field theory and string theory. The generalisation of the notion of instantons to higher dimensions has been first proposed by [6]. In particular, instantons can be defined on any manifold with a $G$-structure. Suppose $G=\mathrm{U}(m)$, such that the (compact) $2 m$-dimensional manifold is endowed with a Kähler structure. In this case, the instanton equations are known as Hermitian Yang-Mills equations and have a deep geometric interpretation in form of the Donaldson-Uhlenbeck-Yau theorem [7, 8]. In contrast, for holonomy $G=\operatorname{Sp}(m)$, the $4 m$ dimensional manifold is hyper-Kähler and the notion of instantons on such spaces has been proposed by $[9,10]$. The generalised Ward correspondence [11], which relates quaternionic instantons on a hyper-Kähler space $M^{4 m}$ with some holomorphic vector bundle on the twistor space of $M^{4 m}$, again provides a relation between gauge theory and holomorphic bundles.

Considering compactifications of 10-dimensional heterotic string theory which preserve $\mathcal{N}=1$ supersymmetry in 4 dimensions, one has to satisfy the so-called BPS equations, which contain an instanton equation on the internal $G$-structure manifold. Unfortunately, for compact Calabi-Yau or compact hyper-Kähler spaces, explicit metrics are not known, but one can resort to cone constructions as a testing ground. The underlying base for a hyperKähler cone is a 3-Sasakian space, while a Calabi-Yau cone starts from a Sasaki-Einstein base. Instantons on certain conical extensions of $G$-manifolds have been considered, for instance, in [12-22]. In all the references, the instanton equations have been reduced to a set of matrix equations by a certain equivariant ansatz. The resulting matrix equations for Calabi-Yau cones over arbitrary Sasaki-Einstein manifolds have been discussed in [21] for one choice of boundary conditions. The aim of the present paper is twofold: firstly, to extend the discussion on the Calabi-Yau cones by considering different boundary conditions, which appear to be more physically relevant. Secondly, to generalise and extend this analysis to the matrix equations resulting from the quaternionic instanton equation on hyper-Kähler cones over arbitrary 3-Sasakian manifolds.

Interestingly, the instanton matrix equations resulting from the equivariant reduction can be viewed as generalised Nahm's equations, called Nahm-type equations in [21]. Recalling the prominent role of Nahm's equations and nilpotent orbits for BPS boundary conditions for 4 -dimensional $\mathcal{N}=4$ super Yang-Mills theories [23, 24], the constructions of 4 -dimensional $\mathcal{N}=1$ theories by compactifying 6 -dimensional theories [25, 26] or assigning $1 / 4$ BPS boundary conditions $[27,28]$ on 4 -dimensional $\mathcal{N}=4$ super-Yang-Mills led to the appearance of generalised Nahm's equations. These are in fact dimensional reductions of 6-dimensional Hermitian Yang-Mills equations and the moduli space of the generalised Nahm's equations will be related to orbits of commuting nilpotent pairs.

The outline of the article is as follows: in section 2 we briefly recall the geometry of Sasaki-Einstein and 3-Sasakian spaces as well as their metric cones. Section 3 is devoted to a description of the moduli space of quaternionic instantons, starting with the space of connections, then showing a reformulation of the $\operatorname{Sp}(m)$-instanton moduli space as intersection of various $\mathrm{SU}(2 m)$-moduli spaces. At the end of this section, we specialise to 
an equivariant ansatz which reduces the instanton equation to a set of matrix equations. In section 4 we treat the instanton matrix equations on the Calabi-Yau cone and show that, depending on the boundary conditions, the moduli space relates to different "diagonal" complex coadjoint orbits. In particular, the choice of singular boundary conditions for the generalised Nahm's equations will naturally lead to orbits of tuples of commuting nilpotent elements. In section 5 , we subsequently extend this study to the instantons on hyper-Kähler cones. Finally, section 6 concludes. Appendix A provides some technical details.

\section{Geometry}

In this section we review the definitions and relevant properties of Sasaki-Einstein and 3-Sasakian manifolds as well as the geometric structure of their metric cones. For details we refer to $[17,29-32]$ and the references therein.

Sasaki-Einstein manifolds. Sasaki manifolds are the odd-dimensional analogues of Kähler manifolds in the sense that a Riemannian manifold $\left(M^{2 n+1}, g\right)$ is Sasakian if and only if its metric cone is a Kähler manifold. That is, the metric cone is a complex manifold with closed Kähler form $\Omega(X, Y):=g(X, J Y)$, or, equivalently, a manifold whose holonomy group is contained in the unitary group $\mathrm{U}(n+1)$.

An equivalent definition (see [29]) is that of a manifold which admits a Killing vector field $\xi$ of unit length such that the type- $(1,1)$ vector field $\Phi(X):=\nabla_{X}^{\mathrm{LC}} \xi$ satisfies

$$
\left(\nabla_{X}^{\mathrm{LC}} \Phi\right)(Y)=g(\xi, Y) X-g(X, Y) \xi
$$

for all vector fields $X$ and $Y$ on $M$. The vector field $\xi$ is referred to as characteristic or Reeb vector field, and Sasakian manifolds are a subclass of metric contact structures.

Denoting by $\eta$ the 1 -form dual to $\xi$, one can consider the Reeb foliation along the characteristic vector field, given by the subbundle $\mathcal{D}:=\operatorname{ker}(\eta)$. This yields transverse Kähler spaces of real dimensions $2 n$, and the corresponding Kähler form $\omega$ follows from the relation $\mathrm{d} \eta=2 \omega$.

A Sasaki-Einstein manifold $\left(M^{2 n+1}, g, \xi\right)$ is a Sasakian manifold whose metric is additionally Einstein, which implies that the metric cone is Calabi-Yau, i.e. a Ricci-flat Kähler manifold. The latter is equivalent to a manifold with special holonomy contained in $\mathrm{SU}(n+1) \subset \mathrm{U}(n+1) \subset \mathrm{SO}(2 n+2)$.

3-Sasakian manifolds. A 3-Sasakian manifold is a Riemannian manifold $\left(M^{4 m+3}, g\right)$ of real dimension $4 m+3$ which admits a triplet of Sasaki structures such that their characteristic vector fields $\xi_{\alpha}$ are orthogonal, $g\left(\xi_{\alpha}, \xi_{\beta}\right)=\delta_{\alpha \beta}$, and satisfy the $\mathrm{SU}(2)$ commutation relations,

$$
\left[\xi_{\alpha}, \xi_{\beta}\right]=2 \epsilon_{\alpha \beta}^{\gamma} \xi_{\gamma}
$$

Note that the existence of this triple of characteristic vector fields implies a whole $\mathbb{C} P^{1}$ family of those structures. Moreover, it can be shown that every 3-Sasakian manifold $M^{4 m+3}$ is automatically Einstein and that its structure group is $\operatorname{Sp}(m)$ (see e.g. [29] and 
the references therein). An alternative definition for 3-Sasakian manifolds is that of being a manifold $M^{4 m+3}$ such that the metric cone is hyper-Kähler, i.e. its Riemannian holonomy is contained in $\mathrm{Sp}(m+1)$. Consequently, a prototype of 3-Sasakian manifolds are the homogeneous spaces $\operatorname{Sp}(m+1) / \mathrm{Sp}(m) \cong S^{4 m+3}$, the squashed spheres. The squashed spheres are $\mathrm{SU}(2)$-bundles, while all other homogeneous 3-Sasakian manifolds are $\mathrm{SO}(3)$-bundles over quaternionic spaces (see e.g. [29]). Another well-known example is the seven-dimensional Aloff-Wallach space $X_{1,1} \cong \mathrm{SU}(3) / \mathrm{U}(1)_{1,1}$.

Hyper-Kähler cones. By definition, a manifold is 3-Sasakian if its metric cone is hyperKähler, i.e. it admits a triplet of covariantly constant complex structures $J_{1}, J_{2}$, and $J_{3}$ satisfying the quaternionic relations

$$
J_{\alpha} J_{\beta}=-\delta_{\alpha \beta} \text { id }+\epsilon_{\alpha \beta}^{\gamma} J_{\gamma}, \quad \text { for } \alpha, \beta, \gamma \in\{1,2,3\} .
$$

These complex structures on the cone are, of course, induced by the three Sasaki structures on the underlying 3-Sasakian manifold and give rise to a triplet of Kähler forms

$$
\Omega_{\alpha}(X, Y):=g\left(X, J_{\alpha}(Y)\right), \quad \text { for } \alpha \in\{1,2,3\},
$$

whose components satisfy relations analogous to (2.3).

Notation. For our discussion we apply the notations used in [17]. A 3-Sasakian manifold is then described by an orthonormal frame of 1 -forms $e^{1}, \ldots, e^{4 m+3}$, where $e^{\alpha} \equiv \eta_{\alpha}$ for $\alpha=1,2,3$ are the duals of the characteristic vector fields $\xi_{\alpha}$, and 2 -forms

$$
\begin{aligned}
& \omega_{1}=\sum_{i=1}^{m}\left(e^{4 i} \wedge e^{4 i+1}+e^{4 i+2} \wedge e^{4 i+3}\right), \\
& \omega_{2}=\sum_{i=1}^{m}\left(e^{4 i} \wedge e^{4 i+2}-e^{4 i+1} \wedge e^{4 i+3}\right), \\
& \omega_{3}=\sum_{i=1}^{m}\left(e^{4 i} \wedge e^{4 i+3}+e^{4 i+1} \wedge e^{4 i+2}\right),
\end{aligned}
$$

which are part of the exterior derivatives of $\eta^{\alpha}$ as follows:

$$
\mathrm{d} \eta_{\alpha}=\epsilon_{\alpha}^{\beta \gamma} \eta_{\beta} \wedge \eta_{\gamma}+2 \omega_{\alpha} \quad\left(\Longrightarrow \mathrm{d} \omega_{\alpha}=2 \epsilon_{\alpha}^{\beta \gamma} \eta_{\beta} \wedge \omega_{\gamma}\right)
$$

The metric of the cone $g_{c}$ (or the conformally equivalent cylinder $g_{\text {cyl }}$ ) reads

$$
g_{c}=r^{2} \sum_{\mu=1}^{4 m+3} e^{\mu} \otimes e^{\mu}+\mathrm{d} r \otimes \mathrm{d} r=r^{2} \sum_{\mu=0}^{4 m+3} e^{\mu} \otimes e^{\mu} \equiv r^{2} g_{\mathrm{cyl}},
$$

with the definition $e^{0}:=\mathrm{d} \tau:=\frac{\mathrm{d} r}{r}$. The induced Kähler forms on the cone read

$$
\Omega_{\alpha}=r^{2}\left(\omega_{\alpha}+\frac{1}{2} \epsilon_{\alpha \beta \gamma} e^{\beta \gamma}+\mathrm{d} \tau \wedge e^{\alpha}\right) .
$$


We note that their closure follows from (2.6). Explicitly, we have

$$
\begin{aligned}
& \Omega_{1}=r^{2} \sum_{i=0}^{m}\left(e^{4 i} \wedge e^{4 i+1}+e^{4 i+2} \wedge e^{4 i+3}\right), \\
& \Omega_{2}=r^{2} \sum_{i=0}^{m}\left(e^{4 i} \wedge e^{4 i+2}-e^{4 i+1} \wedge e^{4 i+3}\right), \\
& \Omega_{3}=r^{2} \sum_{i=0}^{m}\left(e^{4 i} \wedge e^{4 i+3}+e^{4 i+1} \wedge e^{4 i+2}\right),
\end{aligned}
$$

where the summation now starts with $i=0$, in contrast to the expressions for $\omega_{\alpha}$ in (2.5). On the tangent space they induce the complex structures ${ }^{1}$ acting on basis vector fields $E_{0}, \ldots, E_{4 m+3}$ as

$$
J_{\alpha} E_{4 i}=-E_{4 i+\alpha} \quad \text { and } \quad J_{\alpha} E_{4 i+\beta}=-\epsilon_{\alpha \beta \gamma} E_{4 i+\gamma} \quad(\alpha \neq \beta)
$$

and similarly the action on the basis 1 -forms reads

$$
J_{\alpha} e^{4 i}=e^{4 i+\alpha} \quad \text { and } \quad J_{\alpha} e^{4 i+\beta}=\epsilon_{\alpha \beta \gamma} e^{4 i+\gamma} \quad(\alpha \neq \beta) .
$$

for $i=0, \ldots m$.

\section{Comments on moduli space of instantons}

Having established the notation, we proceed by a discussion of generic features for $\mathrm{SU}(n)$ and $\operatorname{Sp}(m)$-instantons. First, we consider the space of connections on hyper-Kähler spaces. Next, we provide the equivalent formulation of the $\mathrm{Sp}(m)$-instanton equations as intersection of three HYM instanton equations. Lastly, we introduce the ansatz for the connection on the cone (or conformally equivalent cylinder) over the Sasaki-Einstein or 3-Sasakian base, which reduces the instanton equations to Nahm-type equations.

\subsection{Space of connections over hyper-Kähler spaces}

In this section we describe the space of connections over a hyper-Kähler manifold $M^{4 m}$ and show that it is equipped with a (formal) hyper-Kähler structure, which is induced from $M^{4 m}$. This account is inspired from the analogous implication for the space of connections over Kähler manifolds, for which we refer to [21, 33, 34].

Preliminaries. Suppose $M^{4 m}$ is a (closed) hyper-Kähler manifold of $\operatorname{dim}_{\mathbb{H}}\left(M^{4 m}\right)=m$ and $G$ is a compact matrix group with $\mathfrak{g}=\operatorname{Lie}(G)$. We denote by $P\left(M^{4 m}, G\right)$ a $G$-principal bundle over $M^{4 m}, \operatorname{Int}(P):=P \times_{G} G$ the group bundle, $\operatorname{Ad}(P):=P \times_{G} \mathfrak{g}$ the Lie algebra bundle, and $E:=P \times_{G} F$ an associated vector bundle (with vector space $F$ that carries a representation of $G$ ).

\footnotetext{
${ }^{1}$ They can also be obtained by writing quadruples $\mathbb{X}_{i}:=X_{4 i}+\mathrm{i} X_{4 i+1}+\mathrm{j} X_{4 i+2}+\mathrm{k} X_{4 i+3}$ and letting $J_{1}=I, J_{2}=J$ and $J_{3}=K$ act on them by multiplication with $\mathrm{i}, \mathrm{j}$ and $\mathrm{k}$.
} 
Then $\mathcal{A}$ is a connection 1-form with curvature $\mathcal{F}_{\mathcal{A}}=\mathrm{d} \mathcal{A}+\mathcal{A} \wedge \mathcal{A}$, and $\mathbb{A}(P)$ (and $\mathbb{A}(E)$ ) denotes the space of connections on $P$ (and $E$ ). The gauge group $\widehat{\mathcal{G}}$ can be identified with the global section on $\operatorname{Int}(P)$, i.e.

$$
\begin{aligned}
& \widehat{\mathcal{G}}=\Gamma\left(M^{4 m}, \operatorname{Int}(P)\right), \\
& \mathcal{A} \mapsto \mathcal{A}^{g}:=\operatorname{Ad}\left(g^{-1}\right) \mathcal{A}+g^{-1} \mathrm{~d} g, \quad \text { for } g \in \widehat{\mathcal{G}} .
\end{aligned}
$$

The associated Lie algebra $\widehat{\mathfrak{g}}$ of $\widehat{\mathcal{G}}$ is identified with the global section on $\operatorname{Ad}(P)$, i.e.

$$
\begin{aligned}
\widehat{\mathfrak{g}} & =\Gamma\left(M^{4 m}, \operatorname{Ad}(P)\right), \\
\mathcal{A} & \mapsto \delta \mathcal{A}=\mathrm{d}_{\mathcal{A}} \chi:=\mathrm{d} \chi+[\mathcal{A}, \chi], \quad \text { for } \chi \in \widehat{\mathfrak{g}} .
\end{aligned}
$$

Moreover, $\mathbb{A}(P)$ is an affine space over $\Omega^{1}\left(M^{4 m}, \operatorname{Ad}(P)\right)$; thus, the tangent space $T_{\mathcal{A}} \mathbb{A}$ for any $\mathcal{A} \in \mathbb{A}(P)$ can be canonically identified with $\Omega^{1}\left(M^{4 m}, \operatorname{Ad}(P)\right)$. By assumption, $G \hookrightarrow \mathrm{U}(N)$, for some $N \in \mathbb{N}$; thus, the trace provides an Ad-invariant inner product.

Metric. A Riemannian structure on $\mathbb{A}(P)$ is established via

$$
\boldsymbol{g}_{\mid \mathcal{A}}(X, Y):=\int_{M^{4 m}} \operatorname{tr}(X \wedge \star Y), \quad \text { for } X, Y \in T_{\mathcal{A}} \mathbb{A}
$$

which is symmetric and base-point independent. Moreover, the definition employs the metric structure on the base manifold via the Hodge star $\star$.

Symplectic forms. Similarly, one can define three symplectic structures on $\mathbb{A}(P)$ via

$$
\left(\boldsymbol{\omega}_{\alpha}\right)_{\mid \mathcal{A}}(X, Y):=\int_{M^{4 m}} \operatorname{tr}(X \wedge Y) \wedge \frac{\left(\Omega_{\alpha}\right)^{2 m-1}}{(2 m-1) !}, \quad \text { for } X, Y \in T_{\mathcal{A}} \mathbb{A}, \alpha=1,2,3,
$$

which is skew-symmetric and base-point independent. Again, the entire $\mathbb{C} P^{1}$-worth of symplectic structures of the base manifold transfers to a $\mathbb{C} P^{1}$ of symplectic structures on $\mathbb{A}(P)$. To show that $\boldsymbol{\omega}_{\alpha}$ is non-degenerate one can explicitly verify that

$$
\star J_{\alpha}(Y)=Y \wedge \frac{\left(\Omega_{\alpha}\right)^{2 m-1}}{(2 m-1) !}, \quad \forall \alpha=1,2,3 .
$$

Here $J_{\alpha}$ acts only on the 1-form part of $Y$. Consequently,

$$
\left(\boldsymbol{\omega}_{\alpha}\right)_{\mid \mathcal{A}}(X, Y)=\int_{M^{4 m}} \operatorname{tr}\left(X \wedge \star J_{\alpha}(Y)\right)=\boldsymbol{g}_{\mid \mathcal{A}}\left(X, J_{\alpha}(Y)\right), \quad \text { for } X, Y \in T_{\mathcal{A}} \mathbb{A}, \alpha=1,2,3,
$$

and $\boldsymbol{\omega}_{\alpha}$ is non-degenerate because $\boldsymbol{g}$ is.

Complex structure. Having a Riemannian and three symplectic structures on $\mathbb{A}(P)$ it is tempting to introduce the compatible complex structures $\boldsymbol{J}_{\alpha}$ via

$$
\boldsymbol{\omega}_{\alpha}(\cdot, \cdot)=\boldsymbol{g}\left(\cdot, \boldsymbol{J}_{\alpha}(\cdot)\right) .
$$


It follows from the above that ${ }^{2}$

$$
\boldsymbol{J}_{\alpha}(Y)=J_{\alpha}(Y), \quad \text { for } Y \in T_{\mathcal{A}} \mathbb{A}, \alpha=1,2,3 .
$$

Thus, the three complex structures on $\mathbb{A}(P)$ are base-point independent, are induced from the complex structures on $M^{4 m}$, and, consequently, satisfy the quaternionic algebra.

In summary, $\mathbb{A}(P)$ (and also $\mathbb{A}(E)$ ) is equipped with a (formal) hyper-Kähler structure, inherited from $M^{4 m}$, and a compatible $\widehat{\mathcal{G}}$-action. We will see in a moment that the moduli space of the hyper-Kähler instanton equations can be understood as a hyper-Kähler quotient thereof.

\subsection{Equivalence of $\operatorname{Sp}(m)$-instantons and intersections of HYM instantons}

Let $M^{4 m}$ be a hyper-Kähler manifold with complex structures $J_{\alpha}$ for $\alpha=1,2,3$ satisfying (2.3) and corresponding Kähler forms $\Omega_{\alpha}$, defined via (2.4). One can parametrise a $\mathbb{C} P^{1}$ of complex structures via $s_{\alpha} \in \mathbb{R}, \delta^{\alpha \beta} s_{\alpha} s_{\beta}=1$ such that any complex structure (and corresponding Kähler form) can be written as

$$
J:=s^{\alpha} J_{\alpha}, \quad \Omega:=s^{\alpha} \Omega_{\alpha} .
$$

Consider a connection $\mathcal{A}$ on a complex vector bundle $E$ over $M^{4 m}$. Since $M^{4 m}$ is hyper-Kähler the generic holonomy $\mathrm{SO}(4 m)$ is reduced to $\mathrm{Sp}(m)$, and one has the splitting

$$
\mathfrak{s o}(4 m)=\mathfrak{s p}(m) \oplus \mathfrak{s p}(1) \oplus \mathfrak{k}
$$

Following the definition of instantons on $G$-structure manifolds, ${ }^{3} \operatorname{Sp}(m)$ instantons are defined as connections such that the curvature 2 -form $\mathcal{F}_{\mathcal{A}}$ takes values in the Lie algebra $\mathfrak{s p}(m)$ only, i.e. the instanton equations are equivalent to the vanishing of the $\mathfrak{s p}(1) \oplus \mathfrak{k}$-part of the curvature 2 -form.

According to $[9,10]$, the $\operatorname{Sp}(m)$-instanton equations can be recast as

$$
\mathcal{F}_{J}^{0,2}=0 \quad \text { for all } J
$$

i.e. they can be obtained from the holomorphicity conditions for any complex structure $J$. Recall that for a fixed $J=J_{\alpha}$ the holomorphicity condition only induces the reduction of the holonomy algebra as

$$
\mathfrak{s o}(4 m)=\mathfrak{u}_{\alpha}(2 m) \oplus \mathfrak{P}_{\alpha},
$$

while HYM instantons additionally constrain the $\mathfrak{u}_{\alpha}(1)$ part of the splitting $\mathfrak{u}_{\alpha}(2 m)=$ $\mathfrak{u}_{\alpha}(1) \oplus \mathfrak{s u}_{\alpha}(2 m)$ by imposing the stability-like condition

$$
\Omega_{\alpha}^{\mu \nu} \mathcal{F}_{\mu \nu}=0, \quad \alpha=1,2,3 .
$$

\footnotetext{
${ }^{2}$ Compared to [21], we consider $\boldsymbol{J}=-\boldsymbol{J}_{\text {can }}$, where $\boldsymbol{J}_{\text {can }}$ is the canonical complex structure defined via $\boldsymbol{\omega}(\cdot, \cdot)=\boldsymbol{g}\left(\boldsymbol{J}_{\mathrm{can}}(\cdot), \cdot\right)$ on $\mathbb{A}(P)$.

${ }^{3}$ Equivalently, one can define $\mathrm{Sp}(m)$-instantons in terms of a generalised self-duality condition; for details, see $[17]$.
} 
However, satisfying the holomorphicity condition (3.11) for any $J$ already implies the stability-like conditions, as it is shown e.g. in section 4.5 of [35]. It can be also seen in the explicit instanton equations we use for the discussion in section 5.1.

Hence, it is justified to consider the moduli space of $\operatorname{Sp}(m)$-instantons as the intersection

$$
\mathcal{M}_{\mathrm{Sp}(m)}=\mathcal{M}_{\mathrm{SU}_{1}(2 m)} \cap \mathcal{M}_{\mathrm{SU}_{2}(2 m)} \cap \mathcal{M}_{\mathrm{SU}_{3}(2 m)}=\bigcap_{J} \mathcal{M}_{\mathrm{SU}_{J}(2 m)} .
$$

\subsection{Quaternionic instantons}

As shown in the previous section, we can understand the $\operatorname{Sp}(m)$-instanton conditions on $E \rightarrow M^{4 m}$ as

$$
\left.\mathcal{F}_{\alpha}^{(0,2)}=0 \quad \text { and } \quad \Omega_{\alpha}\right\lrcorner \mathcal{F}_{\alpha}=0 \quad \forall \alpha=1,2,3
$$

As explained, for instance, in [21], the condition $\mathcal{F}_{\alpha}^{(0,2)}=0$ introduces a holomorphic structure on the vector bundle $E$. Since we have three holomorphic structures arising, the bundle becomes tri-holomorphic. Denote the space of tri-holomorphic connections as

$$
\mathbb{A}^{\text {holo }}(E)=\left\{\mathcal{A} \in \mathbb{A}(E) \mid \mathcal{F}_{\alpha}^{(0,2)}=0, \forall \alpha=1,2,3\right\} .
$$

We expect that $\mathbb{A}^{\text {holo }}(E)$ is equipped with a hyper-Kähler structure by restriction from $\mathbb{A}(E)$ and has a compatible action of $\widehat{\mathcal{G}}$.

On $\mathbb{A}^{\text {holo }}(E)$, the three remaining equations $\left.\Omega_{\alpha}\right\lrcorner \mathcal{F}_{\alpha}=0$ are understood as triplet of moment maps $\mu_{\alpha}$ for the gauge group. The proof of the statement is a generalisation of [33] and has been shown in [21] for the Kähler case. Since the arguments are identical, we refrain from repeating them here.

It is, however, important to realise that the case of non-compact hyper-Kähler cones requires one to consider the framed gauge group $\widehat{\mathcal{G}}_{0}$ for the moment maps to be well-defined.

Thus, we presume that the moduli space of hyper-Kähler instantons can be expressed as (trivial) hyper-Kähler quotient

$$
\mathcal{M}_{\mathrm{Sp}(m)}=\left\{\mathcal{A} \in \mathbb{A}^{\text {holo }}(E) \mid \mu_{\alpha}=0, \forall \alpha=1,2,3\right\} / \widehat{\mathcal{G}}=\mathbb{A}^{\text {holo }}(E) / \widehat{\mathcal{G}} .
$$

The arguments presented earlier imply that it is a trivial quotient in the sense that the moment map conditions are already satisfied on all of $\mathbb{A}^{\text {holo }}(E)$. However, the consequence remains true; $\mathcal{M}_{\mathrm{Sp}(m)}$ is itself a hyper-Kähler space.

\subsection{Ansatz for equivariant instantons}

Before we investigate the instantons on metric cones we briefly describe the set-up, which is based on the approach of [18] and has been thoroughly discussed in [20, 21].

Consider $H=\mathrm{SU}(n)$ or $\mathrm{Sp}(m)$ as closed subgroup of $G=\mathrm{SU}(n+1)$ or $\mathrm{Sp}(m+1)$, respectively. Let $M^{k}(k=2 n+1$ for Sasaki-Einstein and $k=4 m+3$ for 3-Sasakian) be a manifold with $G$-structure together with a canonical connection $\Gamma^{P}$ on the tangent bundle, see [17]. The metric cone, by choice of our examples, is a manifold with reduced holomony 
$G \subset \mathrm{SO}(k+1)$. By conformal invariance of the instanton equations, we can equally well consider $\operatorname{Cyl}\left(M^{k}\right)$, which is equipped with a non-integrable $G$-structure. Let $P$ be the principal $G$-bundle of the frame bundle of $\operatorname{Cyl}\left(M^{k}\right)$ which comprises this $G$-structure and associate a complex vector bundle $E \rightarrow \operatorname{Cyl}\left(M^{k}\right)$ of rank $p$. The fibres $E_{x} \cong \mathbb{C}^{p}$ are equipped with a Hermitian form.

Thus, the connection 1 -from associated to any $\mathcal{A}$ is a $\mathfrak{g}$-valued 1-form on $\operatorname{Cyl}\left(M^{k}\right)$. We consider an ansatz of the form

$$
\mathcal{A}=\widehat{\Gamma}^{P}+X
$$

with $\widehat{\Gamma}^{P}$ denoting the lifted $\mathfrak{h}$-valued connection on $E$ obtained from $\Gamma^{P}$. On a patch $\mathcal{U} \subset \operatorname{Cyl}\left(M^{k}\right)$ with a basis of 1 -forms $\left(e^{0},\left\{e^{\mu}\right\}_{\mu=1}^{k}\right)$ we can describe $X$ via

$$
X_{\mathcal{U}}=X_{0} \otimes e^{0}+X_{\mu} \otimes e^{\mu},
$$

with $X_{\mu \mid x} \in \operatorname{End}\left(\mathbb{C}^{p}\right)$ for $x \in \mathcal{U}$. It is customary to eliminate $X_{0}$ by a suitable gauge transformation - called temporal gauge - but there is no need to do this.

So far, this is just a particular way of rewriting a generic connection. However, we further restrict to connections for which the endomorphisms-valued functions $X_{\mu}$, firstly, depend only on the cone / cylinder coordinate, and, secondly, satisfy an equivariance condition. Since $H$ is a closed subgroup of $G$ one has the $H$-invariant decomposition

$$
\operatorname{span}\left\langle I_{M}\right\rangle \equiv \mathfrak{g}=\mathfrak{h} \oplus \mathfrak{m} \equiv \operatorname{span}\left\langle I_{j}\right\rangle \oplus \operatorname{span}\left\langle I_{\mu}\right\rangle .
$$

Denote by $\widehat{I}_{M}$ the generators in the representation on the fibres of $E_{x} \cong \mathbb{C}^{p}$. The generators satisfy the following commutation relations:

$$
\left[\widehat{I}_{j}, \widehat{I}_{k}\right]=f_{j k}{ }^{l} \widehat{I}_{l}, \quad\left[\widehat{I}_{j}, \widehat{I}_{\mu}\right]=f_{j \mu}{ }^{\nu} \widehat{I}_{\nu}, \quad\left[\widehat{I}_{\mu}, \widehat{I}_{\nu}\right]=f_{\mu \nu}{ }^{j} \widehat{I}_{j}+f_{\mu \nu}{ }^{\sigma} \widehat{I}_{\sigma} .
$$

Then the equivariance conditions read [18]

$$
\left[\widehat{I}_{j}, X_{\mu}\right]=f_{j \mu}{ }^{\nu} X_{\nu}, \quad\left[\widehat{I}_{j}, X_{0}\right]=0 .
$$

These conditions can be satisfied, for instance, by choosing the matrix-valued functions $X_{\mu}$ proportional to the generators spanning $\mathfrak{m}$, i.e. $X_{\mu}=\lambda_{\mu}(r) \widehat{I}_{\mu}$, so that the instanton equations reduce to equations on the scalar functions $\lambda_{\mu}(r)$ only. This approach has been pursued in the constructions of instantons in various settings, see for instance [18-20,22]. Solving the equivariance condition more generally leads to quiver gauge theories [35-40] that depend on the chosen manifold. For the moment, we suppose that one has implemented the equivariance conditions and is left with the relevant instanton equations. We comment on the equivariance condition in section 5.3. As a remark, not imposing (3.21) amounts to dimensional reduction instead of an equivariant reduction, which is legitimate by itself.

In summary, we search for connections satisfying (3.21) and the instanton equations simultaneously. For this ansatz, the gauge group (3.1) reduces to ${ }^{4}$

$$
\widehat{\mathcal{G}}=\{g: \mathbb{R} \rightarrow \mathrm{U}(p)\},
$$

\footnotetext{
${ }^{4}$ Including the equivariance at this stage would imply the decomposition of the gauge group $\{g: \mathbb{R} \rightarrow$ $\left.\prod_{k} \mathrm{U}\left(V_{k}\right)\right\}$ following the decomposition of the endomorphisms space $\left.\operatorname{End}\left(\mathbb{C}^{p}\right)\right|_{H}=\oplus_{k} V_{k}$ on the typical fibre $E_{x} \cong \mathbb{C}^{p}$.
} 
which acts on the matrix-valued functions as follows:

$$
X_{\mu} \mapsto X_{\mu}^{g}:=\operatorname{Ad}(g) X_{\mu}, \quad X_{0} \mapsto X_{0}^{g}:=\operatorname{Ad}(g) X_{0}-\frac{1}{2}\left(\frac{\mathrm{d}}{\mathrm{d} t} g\right) g^{-1} .
$$

As mentioned earlier, due to the non-compactness of the metric cone we need to restrict ourself to the framed gauge transformations.

\section{Instantons on Calabi-Yau cones}

In this section we firstly recap the choice of boundary conditions used in [21] and secondly introduce a different class of boundary conditions. This allows to parallel the HYM matrix instanton equations on the Calabi-Yau cone with the two choices of boundary conditions for Nahm's equations treated by Kronheimer in [41, 42].

\subsection{Set-up}

Before exploring the details, we need to recall the set-up of Nahm's equations and the generalised Nahm's equations for Calabi-Yau instantons.

Nahm's equations. As customary, one splits Nahm's equations in a complex equation [43]

and a real equation

$$
\frac{\mathrm{d} \beta}{\mathrm{d} t}+2 \beta+2[\alpha, \beta]=0
$$

$$
\frac{\mathrm{d}}{\mathrm{d} t}\left(\alpha+\alpha^{*}\right)+2\left(\alpha+\alpha^{*}\right)+2\left(\left[\alpha, \alpha^{*}\right]+\left[\beta, \beta^{*}\right]\right)=0,
$$

for $\alpha=\frac{1}{2}\left(A_{0}+i A_{1}\right)$ and $\beta=\frac{1}{2}\left(A_{2}+i A_{3}\right)$. The $A_{j}$ are the components of a connection on a $G$-bundle $P \rightarrow S^{3} \times \mathbb{R}$. The "model" solution, in temporal gauge $A_{0}=0$, is given by [41]

$$
A_{j}=e^{-2 t} \tau_{j}+\sigma_{j}
$$

where $\tau_{j}$ are elements of a Cartan subalgebra of $\mathfrak{g}$ and $\sigma_{j}$ are elements of $\mathfrak{g}$ that commute with the $\tau_{j}$ and which satisfy the $\mathfrak{s u}(2)$ relations. In more detail, the $\sigma_{j}$ are critical points of a gradient flow; hence, they establish a Lie algebra homomorphism $\rho: \mathfrak{s u}(2) \rightarrow \mathfrak{g}$.

Kronheimer considers the two extreme cases: only $\tau_{j}$ in [41] and only $\sigma_{j}$ in [42]. In both cases, the objective has been to establish the hyper-Kähler structure of certain coadjoint orbits of complex Lie groups via the known hyper-Kähler structure of the moduli space of Nahm's equation. The crucial point in the suitable identification lies in the choice of boundary conditions.

From the physics point of view, ${ }^{5}$ the boundary conditions of [42]

$$
\lim _{t \rightarrow \infty} A(t)=0, \quad \lim _{t \rightarrow-\infty} A(t) \in C(\rho)
$$

are most interesting as they realise the correspondence between the instanton moduli space and nilpotent orbits of the complex Lie group $G^{\mathbb{C}}$. Whereas the regular boundary conditions of [41], led to an identification of the moduli space with the maximal semi-simple orbit.

\footnotetext{
${ }^{5}$ We refer to ([23], section 3$)$ for an accessible review.
} 
Matrix instanton equations on Calabi-Yau cone. In temporal gauge, the instanton matrix equations considered in [21], and also in [18], $\operatorname{read}^{6}$

$$
\begin{aligned}
{\left[X_{2 j-1}, X_{2 k-1}\right] } & =\left[X_{2 j}, X_{2 k}\right], & {\left[X_{2 j-1}, X_{2 k}\right] } & =-\left[X_{2 j}, X_{2 k-1}\right], \\
\frac{\mathrm{d}}{\mathrm{d} t} X_{2 j-1}+\frac{n+1}{n} X_{2 j-1} & =\left[X_{2 j}, X_{2 n+1}\right], & \frac{\mathrm{d}}{\mathrm{d} t} X_{2 j}+\frac{n+1}{n} X_{2 j} & =-\left[X_{2 j-1}, X_{2 n+1}\right],
\end{aligned}
$$

for $j, k=1, \ldots, n$ and

$$
\frac{\mathrm{d}}{\mathrm{d} t} X_{2 n+1}+2 n X_{2 n+1}=\sum_{k=1}^{n}\left[X_{2 k-1}, X_{2 k}\right] .
$$

The novel insight, compared to $[18,21]$, is that the appearing matrix differential equations can be written as gradient flow $\frac{\mathrm{d}}{\mathrm{d} t} X=-\nabla \Psi(X)$ for

$$
\Psi\left(X_{\mu}\right):=\frac{n+1}{2 n} \sum_{a=1}^{2 n} \operatorname{tr}\left(X_{a} X_{a}\right)+n \operatorname{tr}\left(X_{2 n+1} X_{2 n+1}\right)-\operatorname{tr}\left(X_{2 n+1} \sum_{k=1}^{n}\left[X_{2 k-1}, X_{2 k}\right]\right),
$$

while the algebraic conditions (4.4a) have to be imposed as additional constraints. Nevertheless, the additional constraints are preserved by the flow; hence, they only need to hold at one $t_{0} \in \mathbb{R}$ in order to hold at any other instance.

This gradient flow formulation is a reflection of the known phenomenon $[15,17,44]$ that the generalised instanton equations, in temporal gauge, on a cylinder over a manifold $M$ are equivalent to the generalised Chern-Simons gradient flow on $M$ subject to additional constraints. For special cases, like 3-manifolds or 7 -manifolds with nearly parallel $G_{2}$ structure, the additional constraints are implied by the gradient flow.

The generic model solution for (4.4) is of the form, see also [45],

$$
X_{a}=e^{-\frac{n+1}{n} t} T_{a}+S_{a}, a=1, \ldots, 2 n, \quad X_{2 n+1}=e^{-2 n t} T_{2 n+1}+S_{2 n+1},
$$

where the $T_{\mu}$, for $\{\mu\}=\{a, 2 n+1\}$, lie in a Cartan subalgebra, $\left[T_{\mu}, S_{\nu}\right]=0$ for all $\mu, \nu$, and the $S_{\mu}$ are critical points of $\Psi$ subject to the algebraic conditions (4.4a).

This can be put in context to the treatment of Nahm's equations: firstly, the regular boundary conditions for the HYM instantons on Calabi-Yau cones of [21] will lead to a diagonal orbit in which the moduli space can be embedded. Secondly, boundary conditions similar to [42] for the HYM instanton equations have not yet been considered. For the Calabi-Yau instantons it is not straightforward to adapt Kronheimer's analysis, because the critical points of $\Psi$, even imposing the additional constraints (4.4a), do not necessarily give rise to a Lie algebra homomorphism. Nevertheless, one could study boundary conditions for which the $S_{\mu}$ define a Lie algebra homomorphism of $\mathfrak{s u}(n+1)$ in $\mathfrak{u}(p)$. This will be the subject of a later section.

For most of the analysis of the next two sections one only requires the form of the complex equations (4.4a)-(4.4b). We can rewrite the complex equations in the complexified

\footnotetext{
${ }^{6}$ We keep the notation of [21] and label the contact direction of the Sasaki-Einstein structure with $\eta=e^{2 n+1}$.
} 
basis and find

$$
\begin{aligned}
\frac{\mathrm{d}}{\mathrm{d} t} Y_{j}+\frac{n+1}{n} Y_{j}+2\left[Y_{n+1}, Y_{j}\right] & =0, \\
{\left[Y_{j}, Y_{k}\right] } & =0,
\end{aligned}
$$

for $j, k=1, \ldots, n$. Moreover, the linear terms in the instanton matrix equations can be eliminated by a suitable rescaling. For the rescaled matrices we use the notation

$$
X_{a}=e^{-\frac{n+1}{n} t} \mathcal{X}_{a}, \quad X_{2 n+1}=e^{-2 n t} \mathcal{X}_{2 n+1}, \quad X_{2 n+2}=e^{-2 n t} \mathcal{X}_{2 n+2},
$$

and analogously for $Y_{j} \mapsto \mathcal{Y}_{j}$. This rescaling is accompanied by a new variable $s:=-\frac{1}{2 n} e^{-2 n t} \in \mathbb{R}^{-}$.

\subsection{Relation to coadjoint orbits}

The boundary conditions considered in [21] are

$$
\exists g_{0} \in \mathrm{U}(p) \text { such that } \forall \mu=1, \ldots, 2 n+1: \lim _{s \rightarrow-\infty} \mathcal{X}_{\mu}(s)=\operatorname{Ad}\left(g_{0}\right) T_{\mu} \text {, }
$$

where the $T_{\mu}$ lie in a Cartan subalgebra of $\mathfrak{s u}(p)$. For simplification, we can require the $T_{\mu}$ to be a regular tuple, i.e. the intersection of the centralisers of the $T_{\mu}$ consists only of the Cartan subalgebra of $\mathfrak{s u}(p)$. Then all of the $S_{\mu}$ have to vanish such that the $T_{\mu}$ alone provide the only model for the behaviour of the $\mathcal{X}_{\mu}$ near $s \rightarrow-\infty$.

Let us denote by $\mathcal{M}_{n}(E)$ the moduli space of solutions to the complex and real equations satisfying the boundary conditions (4.9) (with suitable regularity) as well as the equivariance condition. From the considerations presented in [21], we can establish the following map

$$
\begin{aligned}
\mathcal{M}_{n}(E) & \rightarrow \mathcal{O}_{\operatorname{diag}}\left(\mathcal{Y}_{1}, \ldots, \mathcal{Y}_{n}\right) \\
(\mathcal{Y}, \mathcal{Z}) & \mapsto\left(\mathcal{Y}_{1}(0), \ldots, \mathcal{Y}_{n}(0)\right)
\end{aligned}
$$

where $\mathcal{O}_{\text {diag }}\left(\mathcal{Y}_{1}, \ldots, \mathcal{Y}_{n}\right)$ is defined as follows: the $n$ commuting objects $\mathcal{Y}_{k}$ can be understood as element of $\mathfrak{g l}(p, \mathbb{C}) \otimes \mathbb{C}^{n}$, because the gauge group $\mathrm{GL}(p, \mathbb{C})$ does not act separately on each $\mathcal{Y}_{k}$, but it acts the same on every $\mathcal{Y}_{k}$. In other words, consider $(\mathrm{GL}(p, \mathbb{C}))^{\times n}$ with the diagonal embedding $\operatorname{GL}(p, \mathbb{C}) \hookrightarrow \operatorname{GL}(p, \mathbb{C})^{\times n}$, which gives rise to the relevant action (3.22). Then we see

$$
\begin{aligned}
\mathcal{O}_{\text {diag }}\left(\mathcal{Y}_{1}, \ldots, \mathcal{Y}_{n}\right) & :=\left\{\left(\operatorname{Ad}(g) \mathcal{Y}_{1}(0), \ldots \operatorname{Ad}(g) \mathcal{Y}_{n}(0)\right) \mid g \in \mathrm{GL}(p, \mathbb{C})\right\} \\
& \subset \prod_{j=1}^{n}\left\{\operatorname{Ad}\left(g_{j}\right) \mathcal{Y}_{j}(0) \mid g_{j} \in \mathrm{GL}(p, \mathbb{C})\right\}=\mathcal{O}_{\mathcal{T}_{1}} \times \cdots \times \mathcal{O}_{\mathcal{T}_{n}}
\end{aligned}
$$

where $\mathcal{O}_{\mathcal{T}_{k}}$ denotes the adjoint orbit of $\mathcal{T}_{k}$ in $\mathfrak{g l}(p, \mathbb{C})$. Analogous to [41], the map (4.10) is injective due to the uniqueness of the corresponding solution of the real and complex equations. In contrast, the surjectivity is less clear. By the construction of the local solution ([21], eq. $(3.40))$, one finds that any element of $\mathcal{O}_{\operatorname{diag}}\left(\mathcal{Y}_{1}, \ldots, \mathcal{Y}_{n}\right)$ gives rise to a 
solution of the complex and real equation, but it is unclear if this solution satisfies the required asymptotic.

Moreover, one knows that the orbit of an element $\mathcal{T}_{k}$ of the Cartan subalgebra is of the form $\operatorname{GL}(p, \mathbb{C}) / \operatorname{Stab}\left(\mathcal{T}_{k}\right)$, where $\operatorname{Stab}\left(\mathcal{T}_{k}\right)$ is the maximal torus of $\operatorname{GL}(p, \mathbb{C})$ because each $\mathcal{T}_{k}$ is assumed to be a regular element. The product of the regular semi-simple coadjoint orbits is a complex symplectic manifold. Each orbit is equipped with the Kirillov-KostantSouriau symplectic form and the product thereof gives the symplectic structure on the total space. As a manifold the orbit $\mathcal{O}_{\operatorname{diag}}\left(\mathcal{Y}_{1}, \ldots, \mathcal{Y}_{n}\right)$ is just $\operatorname{GL}(p, \mathbb{C}) / \operatorname{Stab}\left(\mathcal{Y}_{1}(0), \ldots, \mathcal{Y}_{n}(0)\right)$, wherein

$$
\operatorname{Stab}\left(\mathcal{Y}_{1}(0), \ldots, \mathcal{Y}_{n}(0)\right)=\bigcap_{j=1}^{n} \operatorname{Stab}\left(\mathcal{Y}_{j}(0)\right)=\bigcap_{j=1}^{n} \operatorname{Stab}\left(\mathcal{T}_{j}\right)
$$

and the intersection of the stabilisers of the $\mathcal{T}_{j}$ is the complexified maximal torus, by the regularity assumption. Hence, the complex dimension ${ }^{7}$ is

$$
\operatorname{dim}_{\mathbb{C}}\left(\mathcal{O}_{\operatorname{diag}}\left(\mathcal{Y}_{1}, \ldots, \mathcal{Y}_{n}\right)\right)=\operatorname{dim}_{\mathbb{R}}(\mathrm{U}(p))-\operatorname{rk}(\mathrm{U}(p))=p(p-1),
$$

which always is a multiple of 2. The diagonal orbit is also Kähler, as it is a complex submanifold of a (hyper-)Kähler product. Analogous to [41], the map (4.10) is holomorphic such that it describes an embedding of the framed moduli space $\mathcal{M}_{n}(E)$ into the diagonal orbit, which is a finite-dimensional Kähler manifold.

\subsection{Singular boundary conditions}

In addition, we can consider the other extreme case, in which the boundary conditions are determined by the critical points of the gradient flow.

As mentioned earlier, the equations determining a critical point are not sufficient to define a Lie algebra homomorphism, but they are compatible with a Lie algebra homomorphism. Moreover, note that the basis elements of $\mathfrak{m}$ in the decomposition (3.19) are sufficient to generate $\mathfrak{s u}(n+1)$ as algebra, see also appendix A.

Inspired by the boundary conditions chosen in [42], suppose we have two Lie algebra homomorphisms $\rho_{-}$and $\rho_{+}$. Then we consider boundary conditions of the type ${ }^{8}$

$$
\lim _{t \rightarrow-\infty} X(t) \in C\left(\rho_{-}\right), \quad \lim _{t \rightarrow+\infty} X(t)=\rho_{+} .
$$

Here, $C\left(\rho_{-}\right)$consists of all homomorphisms conjugated to $\rho_{-}$under the adjoint action of $\mathrm{U}(p)$.

The treatment of the instanton matrix equations is as in the case of Nahm's equations: firstly, consider the complex equations with the boundary conditions and identify the equivalence classes of complex trajectories. Secondly, show that each solution of the complex equations can be gauge transformed into a solution of the real equation and that this gauge transformation is unique.

\footnotetext{
${ }^{7}$ In fact, as each $\mathcal{T}_{j}$ is a regular pair, each regular semi-simple $\mathcal{O}_{\mathcal{T}_{k}}$ has the same dimension as the diagonal orbit.

${ }^{8}$ The precise formulation is given in (A.7).
} 
We delegate the details of the "complex trajectories", i.e. solutions to the complex equations satisfying the appropriate boundary conditions, to appendix A, while the treatment of the real equation can be taken over from [21]. We find that the equivalence classes of the complex trajectories associated to homomorphisms $\rho_{ \pm}$are parametrised by a nilpotent orbit and, abusing the name, a "transverse slice" as

$$
\mathcal{N}_{\operatorname{diag}}\left(\rho_{-}\right) \cap S_{\operatorname{diag}}\left(\rho_{+}\right)
$$

with

$$
\begin{aligned}
\mathcal{N}_{\text {diag }}\left(\rho_{-}\right) & :=\left\{\left(\xi_{1}, \ldots, \xi_{n}\right) \in \mathfrak{g r}(p, \mathbb{C}) \otimes \mathbb{C}^{n} \mid \operatorname{Ad}_{g}\left(\xi_{1}, \ldots, \xi_{n}\right)=\left(F_{1}^{-}, \ldots, F_{n}^{-}\right), g \in \mathrm{GL}(p, \mathbb{C})\right\} \\
& \subset \mathcal{N}\left(F_{1}^{-}\right) \times \ldots \times \mathcal{N}\left(F_{n}^{-}\right)
\end{aligned}
$$

where $\mathcal{N}\left(F_{j}^{-}\right)$is the $\mathrm{GL}(p, \mathbb{C})$ nilpotent orbit of $F_{j}^{-}:=\rho_{-}\left(F_{j}\right)$, with $F_{j}$ defined in (A.6). Also, we have defined

$$
S_{\text {diag }}\left(\rho_{+}\right):=\left(F_{1}^{+}, \ldots, F_{n}^{+}\right)+z\left(E_{1}^{+}\right) \times \ldots \times z\left(E_{n}^{+}\right) \subset(\mathfrak{g l}(p, \mathbb{C}))^{\times n} .
$$

Compared to the corresponding expressions in [42] the orbit of the (unique) nilpotent element $Y$ in the case of $\mathrm{SU}(2)$ had to be replaced by a diagonal ${ }^{9}$ orbit of an $n$-tuple of commuting nilpotent elements $F_{j}$. One can assign a notion of nilpotency to this diagonal orbit either naively in the sense that for $\left(\xi_{1}, \ldots, \xi_{n}\right) \in \mathcal{N}_{\text {diag }}\left(\rho_{-}\right)$

$$
\left(\xi_{1}, \ldots, \xi_{n}\right)^{k} \equiv\left(\xi_{1}^{k}, \ldots, \xi_{n}^{k}\right)=(0, \ldots, 0)
$$

for sufficiently large $k$ since each $\xi_{j}$ is nilpotent, or on more general grounds in the context of nilpotent pairs, which we comment on below in section 4.4. Similarly, the expression for the "transverse slice" $\mathcal{S}$ is adapted by considering $n$-tuples of nilpotent elements and the centralisers of the elements of $E_{j}^{+}:=\rho_{+}\left(E_{j}\right)$ that generalise the matrix $X$ in Kronheimer's discussion.

Following ([21], appendix A.4), the treatment of the real equation reduces to two statements: (i) for every complex trajectory there exists a gauge transformation such that the real equation holds, and (ii) equivalent complex trajectories, both satisfying the real equation, are related by a gauge transformation. Denote by $\widetilde{\mathcal{M}}_{n}(E)$ the space of solutions to the instanton matrix equations satisfying (4.14), then we obtain the map

$$
\begin{aligned}
\widetilde{\mathcal{M}}_{n}(E) & \rightarrow \mathcal{N}_{\text {diag }}\left(\rho_{-}\right) \cap S_{\text {diag }}\left(\rho_{+}\right) \\
\left(Y_{j}, Y_{n+1}\right) & \mapsto\left(Y_{1}(0), \ldots, Y_{n}(0)\right),
\end{aligned}
$$

which is clearly injective due to existence and uniqueness of the gauge transformation that renders a complex trajectory into a solution of the real equation. Again, surjectivity is not clear.

If the representation $\rho_{+}$is the trivial representation, then $S_{\text {diag }}\left(\rho_{+}\right)$is all of $(\mathfrak{g l}(p, \mathbb{C}))^{\times n}$ such that the moduli space coincides with the diagonal orbit $\mathcal{N}_{\operatorname{diag}}\left(\rho_{-}\right)$. Note that this

\footnotetext{
${ }^{9}$ It has to be diagonal due to the gauge transformations (3.23) that act with the same group element on all $\mathcal{Y}_{j}$.
} 
implies that the connection $\mathcal{A}$ reduces to the (lifted) canonical connection $\Gamma^{P}$ as $t \rightarrow+\infty$. Consequently, the analogous boundary conditions to (4.3), i.e.

$$
\lim _{t \rightarrow-\infty} X(t) \in C\left(\rho_{-}\right), \quad \lim _{t \rightarrow \infty} X(t)=0,
$$

lead to an embedding of $\widetilde{\mathcal{M}}_{n}(E)$ into (the closure of) a diagonal orbit of an $n$-tuple of commuting nilpotent elements of the complex group.

Moreover, consider the tangent bundle and suppose $\rho_{+}$corresponds to the standard representation of $\mathfrak{s u}(n+1)$ on $E_{\mid p} \cong \mathbb{C}^{n+1}$. Then the corresponding boundary condition reduces the connection $\mathcal{A}$ to the Levi-Civita connection $\nabla^{\mathrm{LC}}$ as $t \rightarrow+\infty$. Thus, on the tangent bundle an instanton solution interpolates between the Levi-Civita connection and the canonical connection, which is consistent with the findings of [17].

Finally, for $\rho_{+}$trivial, one observes that the equivariance conditions (3.21) are certainly compatible with both Lie algebra homomorphisms $\rho_{ \pm}$, provided the $\widehat{I}_{i}$ are the images under $\rho_{-}$of the $\mathfrak{s u}(n)$ generators.

\subsection{Nilpotent pairs and generalised Nahm's equations}

The set-up we have encountered for the singular boundary conditions has close cousins on both sides: mathematics and physics.

Nilpotent pairs. Ginzburg introduced the "doubles" of nilpotent orbits for semi-simple Lie algebras $\mathfrak{g}$ in [46] and has initiated the study of their remarkable properties, which have been further investigated [47-49].

Roughly, a nilpotent pair $e=\left(e_{1}, e_{2}\right) \in \mathfrak{g} \times \mathfrak{g}$ satisfies (i) $\left[e_{1}, e_{2}\right]=0$ and (ii) for an $\left(t_{1}, t_{2}\right) \in \mathbb{C}^{*} \times \mathbb{C}^{*}$, there exists $g=g\left(t_{1}, t_{2}\right) \in G$ such that $\left(t_{1} e_{1}, t_{2} e_{2}\right)=\left(\operatorname{Ad}_{g}\left(e_{1}\right), \operatorname{Ad}_{g}\left(e_{2}\right)\right)$. By this definition, such a pair consists of two commutating nilpotent elements $e_{1}$ and $e_{2}$, but the converse does not necessarily hold.

As noted before, (4.16) is indeed an $n$-tuple of commuting nilpotent elements $F_{j}^{-}$, see also appendix A. Hence, we can view it as an example of a natural generalisation to something like "nilpotent tuples". However, a deeper study of these is beyond the scope of this work.

Generalised Nahm's equations. There exist several generalisations of Nahm's equations in the literature. The one that matches our case is the generalisation considered in [25-28], which contains two copies of Nahm's equations (4.1) coupled by the same $\alpha$.

In $[25,26]$ the construction of 4 -dimensional $\mathcal{N}=1$ theories from 6 -dimensional theories with $\mathcal{N}=(2,0)$ or $(1,0)$ compactified on a Riemann surface with punctures has been studied. Inspired from the dominant role of Hitchin equations in 6 -dimensional $\mathcal{N}=(2,0)$ compactifications to 4 -dimensional $\mathcal{N}=2$ theories, the author of [25] proposed generalised Hitchin equations for $\mathcal{N}=1$ compactifications, from which generalised Nahm's equations have been deduced by reduction. Unsurprisingly, all these generalised equations appear as reductions from the 6-dimensional HYM equations. Moreover, both equations are important for the space of supersymmetric vacua as the moduli space of the generalised Hitchin 
equations describes the Coulomb branch, while the generalised Nahm's equations account for the Higgs branch.

Similarly, in $[27,28] 1 / 4$ BPS boundary conditions for 4-dimensional $\mathcal{N}=4$ super Yang-Mills theory have been studied, which again result in 4-dimensional $\mathcal{N}=1$ theories. Recalling the seminal work by Gaiotto and Witten [23, 24], the study of BPS boundary conditions for $\mathcal{N}=4$ shed light on the importance of nilpotent orbits via Nahm's equations. In the case of $1 / 4$ BPS boundary conditions, generalised Nahm's equations appeared in the very same fashion, and resulting moduli spaces have to be seen in the context of nilpotent pairs. A first account of the moduli space of generalised Nahm's equations has been given in [28] from the GIT quotient perspective.

These instances of generalised Nahm's (and even generalised Hitchin) equations agree with our set-up, because all of them are dimensional reductions of HYM instanton equations on higher dimensional spaces. Our resulting Nahm-type instanton matrix equations arise from an equivariant reduction, which includes the dimensional reduction. Hence, studying the new boundary conditions (4.14) extends the partial description of [28] and formalises generalised Nahm's equations to higher dimensions. Recall that one of the physical origins of our instanton equations are the BPS equations for heterotic flux compactifications. Therefore, we emphasize the special role of HYM in 6 dimensions as generalised Nahm's equations appear as (i) BPS equations in heterotic compactifications to $\mathcal{N}=1$ in $4 \mathrm{~d}$, (ii) BPS equations for compactifications of 6 -dimensional $\mathcal{N}=(2,0)$ theories on Riemann surfaces, and (iii) as $1 / 4$ BPS boundary conditions for 4 -dimensional $\mathcal{N}=4$ super YangMills theory.

In view of [26], in which solutions to generalised Nahm's equations were studied either by reduction to regular Nahm's equations or by products of independent $\mathrm{SU}(2)$ subalgebras, we have considered the scenario in which the tuple of commuting nilpotent elements stems from the orthogonal complement $\mathfrak{m}$ in the decomposition (3.19). This provides another viable option for finding commuting nilpotent elements.

\section{Instantons on hyper-Kähler cones}

In this section we investigate the hyper-Kähler instanton equations for the equivariant ansatz described in section 3.4.

\subsection{Explicit form of hyper-Kähler instanton equations}

We now derive the explicit instanton equations on the hyper-Kähler cone by evaluating the triplet of Hermitian Yang-Mills equations. Denoting, for a fixed $J=J_{\alpha}$, the holomorphic forms as $\theta^{\alpha}=: e^{a_{1}}-\mathrm{i} e^{a_{2}}$ and $\theta^{\beta}=: e^{b_{1}}-\mathrm{i} e^{b_{2}}$, the holomorphicity condition $\mathcal{F}_{\alpha \beta}=0$ reads in terms of real indices

$$
\mathcal{F}_{a_{1} b_{1}}=\mathcal{F}_{a_{2} b_{2}} \quad \text { and } \quad \mathcal{F}_{a_{1} b_{2}}=-\mathcal{F}_{a_{2} b_{1}}
$$


Using the Kähler forms (2.9) on the metric cone, we obtain from the first one the conditions

$$
\begin{aligned}
\mathcal{F}_{4 i, 4 j+2} & =\mathcal{F}_{4 i+1,4 j+3}, & \mathcal{F}_{4 i, 4 j+3} & =-\mathcal{F}_{4 i+1,4 j+2}, \\
\mathcal{F}_{4 i, 4 j} & =\mathcal{F}_{4 i+1,4 j+1}, & \mathcal{F}_{4 i, 4 j+1} & =-\mathcal{F}_{4 i+1,4 j}, \\
\mathcal{F}_{4 i+2,4 j+2} & =\mathcal{F}_{4 i+3,4 j+3}, & \mathcal{F}_{4 i+2,4 j+3} & =-\mathcal{F}_{4 i+3,4 j+2},
\end{aligned}
$$

while the second one yields

$$
\begin{aligned}
\mathcal{F}_{4 i, 4 j+3} & =\mathcal{F}_{4 i+2,4 j+1}, & \mathcal{F}_{4 i, 4 j+1} & =-\mathcal{F}_{4 i+2,4 j+3}, \\
\mathcal{F}_{4 i, 4 j} & =\mathcal{F}_{4 i+2,4 j+2}, & \mathcal{F}_{4 i, 4 j+2} & =-\mathcal{F}_{4 i+2,4 j}, \\
\mathcal{F}_{4 i+3,4 j+3} & =\mathcal{F}_{4 i+1,4 j+1}, & \mathcal{F}_{4 i+3,4 j+1} & =-\mathcal{F}_{4 i+1,4 j+3},
\end{aligned}
$$

and the third Kähler form $\Omega_{3}$ leads to

$$
\begin{aligned}
\mathcal{F}_{4 i, 4 j+1} & =\mathcal{F}_{4 i+3,4 j+2}, & \mathcal{F}_{4 i, 4 j+2} & =-\mathcal{F}_{4 i+3,4 j+1}, \\
\mathcal{F}_{4 i, 4 j} & =\mathcal{F}_{4 i+3,4 j+3}, & \mathcal{F}_{4 i, 4 j+3} & =-\mathcal{F}_{4 i+3,4 j}, \\
\mathcal{F}_{4 i+1,4 j+1} & =\mathcal{F}_{4 i+2,4 j+2}, & \mathcal{F}_{4 i+1,4 j+2} & =-\mathcal{F}_{4 i+2,4 j+1}
\end{aligned}
$$

Note that the holomorphicity conditions with respect to any two of them already imply the third set of conditions. This is not surprising because the characteristic vector fields of two orthogonal Sasaki-structures induce a (unique) third one and therefore a 3-Sasakian structure, see ([32], Chapter 4 Lemma 6). Moreover, adding the relevant equations for $i=j$ one indeed recovers the three stability conditions.

Matrix equations. The canonical connection $\widehat{\Gamma}^{P}$ of [17] is by construction an instanton and the equivariance condition (3.21) ensures that there are no mixed curvature terms, so that the matrices $X_{\mu}$ have to satisfy the instanton equations separately. For convenience, we set $X_{0}=0$ in this paragraph. Following ([17], eq. (4.28)), a suitable choice of structure constants is given by

$$
f_{\beta \gamma}^{\alpha}=-2 \epsilon_{\beta \gamma}^{\alpha}, \quad f_{a b}^{\alpha}=-2 \omega_{a b}^{\alpha}, \quad f_{\alpha b}^{a}=\omega_{a b}^{\alpha} .
$$

Then one obtains from $i=j=0$ in the instanton conditions (5.2) the flow equations for the triplet of matrices accompanying the contact forms $e^{\alpha}$,

$$
\dot{X}_{\alpha}=-2 X_{\alpha}-\frac{1}{2} \epsilon_{\alpha \beta \gamma}\left[X_{\beta}, X_{\gamma}\right]
$$

with $\epsilon_{123}=1$. Setting $i=0$ or $j=0$ yields the flow equations for the other matrices,

$$
\begin{aligned}
& \dot{X}_{4 j}=-X_{4 j}+\left[X_{1}, X_{4 j+1}\right]=-X_{4 j}+\left[X_{2}, X_{4 j+2}\right]=-X_{4 j}+\left[X_{3}, X_{4 j+3}\right], \\
& \dot{X}_{4 j+1}=-X_{4 j+1}-\left[X_{1}, X_{4 j}\right]=-X_{4 j+1}-\left[X_{2}, X_{4 j+3}\right]=-X_{4 j+1}+\left[X_{3}, X_{4 j+2}\right] \text {, } \\
& \dot{X}_{4 j+2}=-X_{4 j+2}+\left[X_{1}, X_{4 j+3}\right]=-X_{4 j+2}-\left[X_{2}, X_{4 j}\right]=-X_{4 j+2}-\left[X_{3}, X_{4 j+1}\right] \text {, } \\
& \dot{X}_{4 j+3}=-X_{4 j+3}-\left[X_{1}, X_{4 j+2}\right]=-X_{4 j+3}+\left[X_{2}, X_{4 j+1}\right]=-X_{4 j+3}-\left[X_{3}, X_{4 j}\right] \text {. }
\end{aligned}
$$


These flow equation coincide, of course, with the general result given in (3.27) of [18] as we are applying their approach with the same structure constants. Finally, for $i, j>0$ the instanton equations (5.2) lead to the algebraic relations

$$
\begin{aligned}
& 4 \delta_{i j} X_{1}=-\left[X_{4 i}, X_{4 j+1}\right]-\left[X_{4 i+2}, X_{4 j+3}\right]=-\left[X_{4 i}, X_{4 j+1}\right]-\left[X_{4 j+2}, X_{4 i+3}\right], \\
& 4 \delta_{i j} X_{2}=-\left[X_{4 i}, X_{4 j+2}\right]+\left[X_{4 i+1}, X_{4 j+3}\right]=-\left[X_{4 i}, X_{4 j+2}\right]+\left[X_{4 j+1}, X_{4 i+3}\right], \\
& 4 \delta_{i j} X_{3}=-\left[X_{4 i}, X_{4 j+3}\right]-\left[X_{4 i+1}, X_{4 j+2}\right]=-\left[X_{4 i}, X_{4 j+3}\right]-\left[X_{4 j+1}, X_{4 i+2}\right],
\end{aligned}
$$

and

$$
\begin{array}{ll}
{\left[X_{4 i}, X_{4 j+1}\right]=\left[X_{4 j}, X_{4 i+1}\right],} & {\left[X_{4 i+2}, X_{4 j+3}\right]=\left[X_{4 j+2}, X_{4 i+3}\right],} \\
{\left[X_{4 i}, X_{4 j+2}\right]=\left[X_{4 j}, X_{4 i+2}\right],} & {\left[X_{4 i+1}, X_{4 j+3}\right]=\left[X_{4 j+1}, X_{4 i+3}\right],} \\
{\left[X_{4 i}, X_{4 j+3}\right]=\left[X_{4 j}, X_{4 i+3}\right],} & {\left[X_{4 i+1}, X_{4 j+2}\right]=\left[X_{4 j+1}, X_{4 i+2}\right],}
\end{array}
$$

as well as

$$
\left[X_{4 i}, X_{4 j}\right]=\left[X_{4 i+1}, X_{4 j+1}\right]=\left[X_{4 i+2}, X_{4 j+2}\right]=\left[X_{4 i+3}, X_{4 j+3}\right]
$$

While for $m=0$ the system reduces to the well-known equations on the $\mathrm{SU}(2)$-triplet of contact forms (5.4), for any positive $m$ the system gets significantly more complicated due to the occurrence of the non-trivial algebraic relations (5.6). In particular, the three matrices $X_{\alpha}$ can be expressed as commutators of the other matrices, so that the flow equations for $X_{a}$ are actually cubic in the endomorphisms. Moreover, by virtue of these algebraic conditions, the flow equations for $X_{\alpha}, \alpha=1,2,3$, follow from the flow equations (5.5) of the other matrices.

We will comment on this algebraic behaviour, different from that of instanton equations for a single Sasaki-Einstein structure as in [21], in more detail in the following section.

\subsection{Single HYM moduli space}

The discussion of section 3.2 has shown that $\mathrm{Sp}(m)$-instantons on hyper-Kähler cones over 3-Sasakian manifolds can be described as the intersection of the holomorphicity conditions with respect to the $\mathbb{C} P^{1}$ family of Sasaki-Einstein structures. As shown in the previous section, it is even sufficient to consider only the intersection of the HYM equations with respect to two orthogonal Sasaki-Einstein structures.

Therefore, we give a description of such a HYM space here, commenting also on the differences compared to [21] due to the different algebraic conditions. Without loss of generality, let us specialise to $\Omega=\Omega_{3}$ in the following.

Conceptually, we need to adjust the setting compared to section 4: the starting point $\Gamma^{P}$ is the canonical connection for the $\mathrm{Sp}(m)$-structure on the base, which is an $\mathrm{SU}(2 m+1)$ instanton due to $\mathrm{Sp}(m) \subset \mathrm{SU}(2 m) \subset \mathrm{SU}(2 m+1)$. Nevertheless, the more "natural" starting point would have been the canonical instanton in the sense of [17] associated to the SasakiEinstein $\mathrm{SU}(2 m+1)$-structure, as it has been used for the Calabi-Yau cones in [21]. 
Holomorphicity condition. The holomorphicity conditions of the HYM equations for $\Omega_{3}$ yield the differential equations

$$
\begin{aligned}
\dot{X}_{1} & =-\left[X_{2}, X_{3}\right]-2 X_{1} & \dot{X}_{2} & =-\left[X_{3}, X_{1}\right]-2 X_{2}, \\
\dot{X}_{4 i+1} & =\left[X_{3}, X_{4 i+2}\right]-X_{4 i+1} & \dot{X}_{4 i+2} & =-\left[X_{3}, X_{4 i+1}\right]-X_{4 i+2}, \\
\dot{X}_{4 i} & =\left[X_{3}, X_{4 i+3}\right]-X_{4 i} & \dot{X}_{4 i+3} & =-\left[X_{3}, X_{4 i}\right]-X_{4 j+3},
\end{aligned}
$$

together with the algebraic relations (for $i, j>0$ )

$$
\begin{array}{ll}
{\left[X_{1}, X_{4 i+1}\right]=\left[X_{2}, X_{4 i+2}\right]} & {\left[X_{1}, X_{4 i+2}\right]=-\left[X_{2}, X_{4 i+1}\right],} \\
{\left[X_{1}, X_{4 i}\right]=\left[X_{2}, X_{4 i+3}\right],} & {\left[X_{1}, X_{4 i+3}\right]=-\left[X_{2}, X_{4 i}\right] .}
\end{array}
$$

and

$$
\begin{aligned}
4 \delta_{i j} X_{1} & =\left[X_{4 i+3}, X_{4 j+2}\right]-\left[X_{4 i}, X_{4 j+1}\right] & 4 \delta_{i j} X_{2} & =-\left[X_{4 i+3}, X_{4 j+1}\right]-\left[X_{4 i}, X_{4 j+2}\right], \\
0 & =\left[X_{4 i}, X_{4 j}\right]-\left[X_{4 i+3}, X_{4 j+3}\right] & 0 & =\left[X_{4 i}, X_{4 j+3}\right]-\left[X_{4 j}, X_{4 i+3}\right], \\
0 & =\left[X_{4 i+1}, X_{4 j+1}\right]-\left[X_{4 i+2}, X_{4 j+2}\right] & 0 & =\left[X_{4 i+1}, X_{4 j+2}\right]-\left[X_{4 j+1}, X_{4 i+2}\right] .
\end{aligned}
$$

Again, the algebraic conditions combined with the differential equations of $X_{a}$ for $a=4, \ldots, 4 m+3$ imply the differential equations for the endomorphisms $X_{1}$ and $X_{2}$.

Stability-like condition. Evaluating $\left.\Omega_{3}\right\lrcorner \mathcal{F}=0$ for the given form $\Omega_{3}$ and the structure constants (5.3) leads to

$$
\begin{aligned}
-\dot{X}_{3} & =\left[X_{1}, X_{2}\right]+2 X_{3}+\sum_{i=1}^{m}\left(\left[X_{4 i+1}, X_{4 i+2}\right]+2 X_{3}+\left[X_{4 i}, X_{4 i+3}\right]+2 X_{3}\right) \\
& =\left[X_{1}, X_{2}\right]+2(2 m+1) X_{3}+\sum_{i=1}^{m}\left(\left[X_{4 i+1}, X_{4 i+2}\right]+\left[X_{4 i}, X_{4 i+3}\right]\right) .
\end{aligned}
$$

As in the previous discussions of the Nahm-type equations, the flow equations (5.9) will be referred to as complex equations, while the stability-like condition is the real equation.

Gradient flow. The differential equations (5.9) and (5.12) among the instanton matrix equations can be cast as gradient flow equations $\frac{\mathrm{d}}{\mathrm{d} t} X=-\nabla \Phi(X)$ similarly to Nahm's equations [42]. To see this, let $\Phi: \mathfrak{u}(p)^{\times(4 m+3)} \rightarrow \mathbb{R}$ be the function defined as

$$
\begin{aligned}
\Phi\left(X_{\alpha}, X_{a}\right):= & \operatorname{tr}\left(X_{1} X_{1}\right)+\operatorname{tr}\left(X_{2} X_{2}\right)+(2 m+1) \operatorname{tr}\left(X_{3} X_{3}\right)+\frac{1}{2} \sum_{a=4}^{4 m+3} \operatorname{tr}\left(X_{a} X_{a}\right) \\
& +\operatorname{tr}\left(X_{3}\left[X_{1}, X_{2}\right]\right)+\operatorname{tr}\left(X_{3} \sum_{j=1}^{m}\left(\left[X_{4 j+1}, X_{4 j+2}\right]+\left[X_{4 j}, X_{4 j+3}\right]\right)\right) .
\end{aligned}
$$

The algebraic equations (5.10), (5.11) are not part of this system, but they are invariant under the gradient flow. Thus, if they are satisfied at any point $t_{0}$ then they hold throughout the evolution. 
Rewriting of the matrix equations. One can eliminate the linear terms in the instanton matrix equations (5.9) and (5.12) by a suitable rescaling as follows:

$$
X_{\gamma}=\mathrm{e}^{-2(2 m+1) \tau} \mathcal{X}_{\gamma}(\gamma=0,3), \quad X_{\beta}=\mathrm{e}^{-2 \tau} \mathcal{X}_{\beta} \quad(\beta=1,2), \quad \text { and } \quad X_{a}=\mathrm{e}^{-\tau} \mathcal{X}_{a}
$$

for $a=4, \ldots 4 m+3$, which is accompanied by a rescaled cone coordinate

$$
s:=-\frac{1}{2(2 m+1)} \mathrm{e}^{-2(2 m+1) \tau}
$$

In addition, we combine the matrices into the complex fields (defined w.r.t. $J_{3}$ ),

$$
\begin{aligned}
\mathcal{P}_{i} & :=\frac{1}{2}\left(\mathcal{X}_{4 i+1}+\mathrm{i} \mathcal{X}_{4 i+2}\right), & \mathcal{Q}_{i} & :=\frac{1}{2}\left(\mathcal{X}_{4 i}+\mathrm{i} \mathcal{X}_{4 i+3}\right), \\
\mathcal{Y} & :=\frac{1}{2}\left(\mathcal{X}_{1}+\mathrm{i} \mathcal{X}_{2}\right), & \mathcal{Z} & :=\frac{1}{2}\left(\mathcal{X}_{0}+\mathrm{i} \mathcal{X}_{3}\right) .
\end{aligned}
$$

The complex equations then read as follows: the purely algebraic relations (5.10) and (5.11)

$$
\left[\mathcal{P}_{i}, \mathcal{P}_{j}\right]=0=\left[\mathcal{Q}_{i}, \mathcal{Q}_{j}\right], \quad\left[\mathcal{P}_{i}, \mathcal{Y}\right]=0=\left[\mathcal{Q}_{i}, \mathcal{Y}\right], \quad\left[\mathcal{P}_{i}, \mathcal{Q}_{j}\right]=2 \delta_{i j} \mathcal{Y}
$$

for $i, j=1, \ldots m$, which are the commutation relations of a (complexified) Heisenberg algebra. Moreover, the differential equations simplify to

$$
\frac{\mathrm{d}}{\mathrm{d} s} \mathcal{P}_{i}=2\left[\mathcal{P}_{i}, \mathcal{Z}\right], \quad \frac{\mathrm{d}}{\mathrm{d} s} \mathcal{Q}_{i}=2\left[\mathcal{Q}_{i}, \mathcal{Z}\right], \quad \frac{\mathrm{d}}{\mathrm{d} s} \mathcal{Y}=2[\mathcal{Y}, \mathcal{Z}]
$$

The real equation becomes

$$
\frac{\mathrm{d}}{\mathrm{d} s}\left(\mathcal{Z}+\mathcal{Z}^{\dagger}\right)+2\left[\mathcal{Z}, \mathcal{Z}^{\dagger}\right]+\lambda_{1}(s)\left[\mathcal{Y}, \mathcal{Y}^{\dagger}\right]+\lambda_{2}(s) \sum_{i=1}^{m}\left(\left[\mathcal{Q}_{i}, \mathcal{Q}_{i}^{\dagger}\right]+\left[\mathcal{P}_{i}, \mathcal{P}_{i}^{\dagger}\right]\right)=0
$$

with the functions

$$
\lambda_{1}(s):=\left(-\frac{1}{2(2 m+1) s}\right)^{\frac{2(2 m+1)-2}{2 m+1}} \text { and } \quad \lambda_{2}(s):=\left(-\frac{1}{2(2 m+1) s}\right)^{\frac{2(2 m+1)-1}{2 m+1}} .
$$

Note that the definition of $s$ coincides with that in [21] (recalling $n=2 m+1$ in that notation), but that the functions $\lambda$ differ because in the hyper-Kähler case only the rescaling of $X_{3}$ depends on $m$ while the other factors of $\tau$ in (5.14) are the same for all dimensions $m$.

Similarly to the remarks in section 4.4 , one may think of the above equations as $m$ copies of the same system, which all have the same matrix $\mathcal{Y}$. This, of course, just reflects the geometry of the hyper-Kähler manifold as consisting of quaternionic "blocks" on which the defining structures act. 
Preliminaries. Just to be explicit, we recall the gauge transformations for complex matrix equations. The real and the complex group of gauge transformations (with respect to $J_{3}$ ) are respectively

$$
\widehat{\mathcal{G}}_{\mathrm{HYM}}=\left\{g: \mathbb{R}^{-} \rightarrow \mathrm{SU}(p)\right\}, \quad \widehat{\mathcal{G}}_{\mathrm{HYM}}^{\mathbb{C}}=\left\{g: \mathbb{R}^{-} \rightarrow \mathrm{SL}(p, \mathbb{C})\right\} .
$$

The transformation rules of the complex linear combinations (5.16) are

$$
\begin{aligned}
\mathcal{P}_{j} & \mapsto \mathcal{P}_{j}^{g}:=\operatorname{Ad}(g) \mathcal{P}_{j}, & \mathcal{Q}_{j} & \mapsto \mathcal{Q}_{j}^{g}:=\operatorname{Ad}(g) \mathcal{Q}_{j} \quad \text { for } j=1, \ldots, m, \\
\mathcal{Y} & \mapsto \mathcal{Y}^{g}:=\operatorname{Ad}(g) \mathcal{Y}, & \mathcal{Z} & \mapsto \mathcal{Z}^{g}:=\operatorname{Ad}(g) \mathcal{Z}-\frac{1}{2}\left(\frac{\mathrm{d}}{\mathrm{d} s} g\right) g^{-1} .
\end{aligned}
$$

We emphasize that the complex equations are invariant under the complex gauge transformations, while the real equation is only invariant under the real gauge transformations.

Formulation of boundary conditions. As discussed in section 4, the generic model solution is of the form

$$
\begin{aligned}
X_{a} & =\mathrm{e}^{-\tau} \mathcal{T}_{a}+\mathcal{S}_{a}, & a & =4, \ldots 4 m+3, \\
X_{\beta} & =\mathrm{e}^{-2 \tau} \mathcal{T}_{\beta}+\mathcal{S}_{\beta}, & \beta & =1,2, \\
X_{3} & =\mathrm{e}^{-2(2 m+1) \tau} \mathcal{T}_{3}+\mathcal{S}_{3}, \text { and } & X_{0} & =0
\end{aligned}
$$

where the $\mathcal{T}$ are a solution to

$$
\begin{aligned}
{\left[\mathcal{P}_{i}, \mathcal{P}_{j}\right] } & =0=\left[\mathcal{Q}_{i}, \mathcal{Q}_{j}\right], \\
{\left[\mathcal{P}_{i}, \mathcal{Y}\right] } & =0=\left[\mathcal{Q}_{i}, \mathcal{Y}\right], \quad\left[\mathcal{P}_{i}, \mathcal{Q}_{j}\right]=2 \delta_{i j} \mathcal{Y}, \\
{\left[\mathcal{P}_{i}, \mathcal{Z}\right] } & =0=\left[\mathcal{Q}_{i}, \mathcal{Z}\right]=[\mathcal{Y}, \mathcal{Z}],
\end{aligned}
$$

wherein the complex linear combinations (5.16) are formed out of the $\mathcal{T}$.

The obvious observation is $\mathcal{Z}$ commutes with every other matrix. Next, $\mathcal{P}_{i}$ and $\mathcal{Q}_{j}$ commute with each other and among themselves; thus, resembling the complexified algebra of $\mathbb{R}^{2 m}$. However, $\mathcal{Y}$ introduces a central extension, which renders the algebra spanned by $\mathcal{P}_{i}, \mathcal{Q}_{j}, \mathcal{Y}$ into a complexified Heisenberg algebra $H_{m}^{\mathbb{C}}$. The solution space to (5.23) is not empty, because the choice $\mathcal{Y} \equiv 0$ allows all other generators to be chosen from a Cartan subalgebra of $\mathfrak{g l}(p, \mathbb{C})$.

Next, the matrices $\mathcal{S}$ commute with all $\mathcal{T}$ and are critical points of $\Phi$, see (5.13), subject to the additional constraints $(5.10),(5.11)$. In detail, $\mathcal{S}$ need to satisfy the following commutation relations:

$$
\begin{aligned}
{\left[\mathcal{S}_{2}, \mathcal{S}_{3}\right] } & =-2 \mathcal{S}_{1}, & {\left[\mathcal{S}_{3}, \mathcal{S}_{1}\right] } & =-2 \mathcal{S}_{2}, \\
{\left[\mathcal{S}_{3}, \mathcal{S}_{4 i+2}\right] } & =\mathcal{S}_{4 i+1}, & {\left[\mathcal{S}_{3}, \mathcal{S}_{4 i+1}\right] } & =-\mathcal{S}_{4 i+2}, \\
{\left[\mathcal{S}_{3}, \mathcal{S}_{4 i+3}\right] } & =\mathcal{S}_{4 i}, & {\left[\mathcal{S}_{3}, \mathcal{S}_{4 i}\right] } & =-\mathcal{S}_{4 j+3}, \\
{\left[\mathcal{S}_{1}, \mathcal{S}_{4 i+1}\right] } & =\left[\mathcal{S}_{2}, \mathcal{S}_{4 i+2}\right], & {\left[\mathcal{S}_{1}, \mathcal{S}_{4 i+2}\right] } & =-\left[\mathcal{S}_{2}, \mathcal{S}_{4 i+1}\right], \\
{\left[\mathcal{S}_{1}, \mathcal{S}_{4 i}\right] } & =\left[\mathcal{S}_{2}, \mathcal{S}_{4 i+3}\right], & {\left[\mathcal{S}_{1}, \mathcal{S}_{4 i+3}\right] } & =-\left[\mathcal{S}_{2}, \mathcal{S}_{4 i}\right],
\end{aligned}
$$


and

$$
\begin{aligned}
4 \delta_{i j} \mathcal{S}_{1} & =\left[\mathcal{S}_{4 i+3}, \mathcal{S}_{4 j+2}\right]-\left[\mathcal{S}_{4 i}, \mathcal{S}_{4 j+1}\right], & 4 \delta_{i j} \mathcal{S}_{2} & =-\left[\mathcal{S}_{4 i+3}, \mathcal{S}_{4 j+1}\right]-\left[\mathcal{S}_{4 i}, \mathcal{S}_{4 j+2}\right] \\
0 & =\left[\mathcal{S}_{4 i}, \mathcal{S}_{4 j}\right]-\left[\mathcal{S}_{4 i+3}, \mathcal{S}_{4 j+3}\right], & 0 & =\left[\mathcal{S}_{4 i}, \mathcal{S}_{4 j+3}\right]-\left[\mathcal{S}_{4 j}, \mathcal{S}_{4 i+3}\right] \\
0 & =\left[\mathcal{S}_{4 i+1}, \mathcal{S}_{4 j+1}\right]-\left[\mathcal{S}_{4 i+2}, \mathcal{S}_{4 j+2}\right], & 0 & =\left[\mathcal{S}_{4 i+1}, \mathcal{S}_{4 j+2}\right]-\left[\mathcal{S}_{4 j+1}, \mathcal{S}_{4 i+2}\right]
\end{aligned}
$$

as well as

$$
0=\left[\mathcal{S}_{1}, \mathcal{S}_{2}\right]+2(2 m+1) \mathcal{S}_{3}+\sum_{i=1}^{m}\left(\left[\mathcal{S}_{4 i+1}, \mathcal{S}_{4 i+2}\right]+\left[\mathcal{S}_{4 i}, \mathcal{S}_{4 i+3}\right]\right)
$$

However, in contrast to the case of Nahm's equations [42] the critical points of $\Phi$ do not necessarily give rise to a Lie algebra homomorphism, unless one considers the trivial case $m=0$, which reduces to the set-up of the original Nahm's equations, of course.

Comparing to $[21,45]$, one could impose that the $\mathcal{T}$ are a regular ${ }^{10}$ tuple, but one would necessarily have to set $\mathcal{Y}=0$. For boundary conditions with non-vanishing $\mathcal{Y}$, regularity cannot be required. Consequently, one cannot dismiss the possibility of having non-trivial $\mathcal{S}$ in the boundary conditions. Therefore, the generic solution to the matrix equations is determined by two tuples $\mathcal{T}$ and $\mathcal{S}$ of matrices in the boundary conditions.

The situation is in analogy to Nahm's equations considered in [41, 42, 50, 51]. While Kronheimer studied the "extreme" cases $\mathcal{T}=0$ or $\mathcal{S}=0$, Biquard and Kovalev considered generic boundary conditions. In all cases, the idea has been to assign suitable boundary conditions to the Nahm equations, for which the moduli space is known to be hyper-Kähler due to Hitchin, and conclude that general coadjoint orbits of complexified Lie groups are hyper-Kähler. Here, we aim for less: learn as much as possible about the HYM matrix instanton equations by generalising this analysis, because the matrix equations exhibit a Nahm-type structure.

In the light of section 4 , we expect that the solutions to the complex equations are classified by a "diagonal" generalisation of a general coadjoint orbit. This is similar to $\mathcal{O}_{\text {diag }}$ of (4.11) and $\mathcal{N}_{\text {diag }}$ of (4.16). The analysis is expected to follow the arguments of [50]. In other words, one first considers the complex equations for suitable boundary conditions. Most arguments from section 4 and [21] still hold, only the local solution has to be adapted. Thus, we expect that the conjugacy classes of the "complex trajectories" can be identified with a suitable orbit. Secondly, the analysis of the real equations remains the same. For our intents and purposes, it therefore suffices to note that the moduli space of the HYM matrix equations has a Kähler structure and is mapped into some finite-dimensional orbit space.

However, despite the formal similarities in the description of a single HYM moduli space contained in the description of the $\operatorname{Sp}(m)$ instantons, one should keep in mind that the intersection (3.14) is very restrictive: since the triplet $X_{\alpha}$ has to transform the same and due to the way they couple to each other in the matrix equations, there is no (obvious) gauge transformation for all Kähler structures simultaneously in the case $m \geq 1$. Therefore, one may need completely new tools, taking into account the SU(2)-symmetry of the fibre and the quaternionic structures of the other matrices explicitly, to describe the generic properties of the moduli space.

\footnotetext{
${ }^{10}$ The intersection of the centralisers of the $T_{\mu}$ consists only of a Cartan subalgebra.
} 


\subsection{Space of equivariant connections}

Due to the choice of structure constants (5.3) and their intimate relationship to the hyperKähler structure forms (2.9), it is not surprising that one can express the equivariance conditions as holomorphic equations in the matrix valued-functions for a given complex structure $\boldsymbol{J}_{\alpha}$. The relation to the complex structure on the metric cone over $M^{4 m+3}$ is established via (3.8).

The exact description of the equivariant connections depends on the concrete 3-Sasakian manifold taken into account, but we review the example of the squashed sevensphere, as studied in [35]. Its equivariance condition requires $\left[\widehat{I}_{j}, X_{\mu}\right]=f_{j \mu}^{\nu} X_{\nu}$, where $j=8,9,10$ labels the generators of the $\mathrm{Sp}(1)$ subgroup. The relevant non-vanishing structure constants are ${ }^{11}$

$$
\begin{aligned}
-f_{85}^{4} & =f_{96}^{4}=f_{10,7}^{4}=1, \quad f_{84}^{5}=f_{97}^{5}=-f_{10,6}^{5}=1 \\
f_{87}^{6} & =-f_{94}^{6}=f_{10,5}^{6}=1, \quad-f_{86}^{7}=-f_{95}^{7}=-f_{10,4}^{7}=1 .
\end{aligned}
$$

and the equivariance conditions therefore read

$$
\begin{aligned}
& {\left[\widehat{I}_{8}, X_{4}\right]=X_{5}, \quad\left[\widehat{I}_{8}, X_{5}\right]=-X_{4}, \quad\left[\widehat{I}_{8}, X_{6}\right]=-X_{7}, \quad\left[\widehat{I}_{8}, X_{7}\right]=X_{6},} \\
& {\left[\widehat{I}_{9}, X_{4}\right]=-X_{6}, \quad\left[\widehat{I}_{9}, X_{5}\right]=-X_{7}, \quad\left[\widehat{I}_{9}, X_{6}\right]=X_{4}, \quad\left[\widehat{I}_{9}, X_{7}\right]=X_{5} \text {, }} \\
& {\left[\widehat{I}_{10}, X_{4}\right]=-X_{7}, \quad\left[\widehat{I}_{10}, X_{5}\right]=X_{6}, \quad\left[\widehat{I}_{10}, X_{6}\right]=-X_{5}, \quad\left[\widehat{I}_{10}, X_{7}\right]=X_{4},}
\end{aligned}
$$

and

$$
\left[\widehat{I}_{j}, X_{\alpha}\right]=0 \quad \forall j=8,9,10, \alpha=1,2,3 .
$$

The complex structures act according to $J_{\alpha}$ in (2.10) on the tangent vectors $\delta X_{\mu}$. Imposing the equivariance condition is compatible with the hyper-Kähler structure given by $J_{\alpha}$ because the equations are invariant. Consider for instance

$$
\left[\widehat{I}_{8}, X_{4}\right]=X_{5} \quad \Rightarrow \quad\left[\widehat{I}_{8}, \delta X_{4}\right]=\delta X_{5}
$$

Applying $J_{1}$ gives us

$$
\left[\widehat{I}_{8}, J_{1}\left(\delta X_{4}\right)\right]=\left[\widehat{I}_{8},-\delta X_{5}\right]=-\left(-\delta X_{4}\right)=J_{1}\left(\delta X_{5}\right)=J_{1}\left[\widehat{I}_{8},\left(\delta X_{4}\right)\right] .
$$

Similarly, we have

$$
\begin{aligned}
& {\left[\widehat{I}_{8}, J_{2}\left(\delta X_{4}\right)\right]=\left[\widehat{I}_{8},-\delta X_{6}\right]=-\left(-\delta X_{7}\right)=J_{2}\left(\delta X_{5}\right)=J_{2}\left[\widehat{I}_{8},\left(\delta X_{4}\right)\right]} \\
& {\left[\widehat{I}_{8}, J_{3}\left(\delta X_{4}\right)\right]=\left[\widehat{I}_{8},-\delta X_{7}\right]=-\delta X_{6}=J_{3}\left(\delta X_{5}\right)=J_{3}\left[\widehat{I}_{8},\left(\delta X_{4}\right)\right] .}
\end{aligned}
$$

Thus, the space of equivariant connections $\mathbb{A}^{\text {equiv }}$ is a tri-holomorphic subspace of $\mathbb{A}^{\text {holo }}$. The remaining question is whether or not the metric or equivalently the symplectic structure is non-degenerate on the vanishing locus of (3.21). It seems difficult to obtain an exact statement for the generic case.

\footnotetext{
${ }^{11}$ By mapping the indices $\left(e^{1}, e^{2}, e^{3}, e^{4}, e^{5}, e^{6}, e^{7}\right) \mapsto\left(e^{4}, e^{5}, e^{6}, e^{7},-e^{2},-e^{3}, e^{1}\right)$ we relate our notation with that of [35].
} 


\section{Summary and conclusions}

In the course of this article we considered higher-dimensional instantons on Calabi-Yau cones and hyper-Kähler cones over arbitrary Sasaki-Einstein and 3-Sasakian manifolds $M^{k}$, respectively. It is known that the instanton moduli space over a (hyper-)Kähler manifold is (hyper-)Kähler, resulting from an infinite-dimensional (hyper-)Kähler quotient. It seems naturally that the subset of invariant connections inherits this property, but the overall situation remains unknown.

In the ansatz (3.18) presented, we restricted the connections to those obtained by extension of the (lifted) canonical connection $\Gamma^{P}$ on $T M^{k}$ by $t$-dependent endomorphismsvalued 1-forms $X_{\mu}(t) \otimes e^{\mu}$ which satisfy an equivariance condition (3.21). For this ansatz we specified the geometric structures on the space of connections.

In section 4 we have significantly extended the study of the Nahm-type instanton matrix equations on Calabi-Yau cones. On the one hand, we extended the discussion of the regular boundary conditions started earlier in [21] by providing details of the relevant diagonal coadjoint orbit (4.11). On the other hand, we have conducted the full treatment of boundary conditions given by Lie algebra homomorphisms. As in the study of 4-dimensional instantons, these boundary conditions seem to be the most physical, as they relate to known instantons, for instance, on the tangent bundle. Similar to Kronheimer's case, the moduli space is related to a "diagonal" nilpotent orbit (4.16) or, equivalently, an orbit of an $n$-tuple of commutating nilpotent elements.

These generalised Nahm's equations appear in the construction of 4-dimensional $\mathcal{N}=1$ theories from heterotic string theory, 6-dimensional gauge theories, or 4-dimensional theories with higher amount of supersymmetry. We complemented the study of their moduli space, started in $[27,28]$, and generalised the system to the reduction obtained from $2 n$ dimensional HYM-equations, $n \geq 3$. In addition, the ansatz taken represents a complementary ansatz compared to [26]. The treatment of generalised Nahm's equations for $n \geq 3$ suggests a close relationship of their moduli space with orbits of $n$-tuples of commuting nilpotent elements, which we proposed as natural extension of nilpotent pairs introduced in [46]. However, since the classification of commuting nilpotent pairs is, up to our knowledge, still an open problem, we refrain from any speculation about nilpotent $n$-tuples.

Using the equivalence between $\operatorname{Sp}(m)$-instantons and a $\mathbb{C} P^{1}$-family of HYM instantons, we described explicitly the system of HYM instanton matrix equations of a single $\mathrm{SU}(2 m)$-structure in section 5. Due to the different starting point $\Gamma^{P}$ on the 3-Sasakian base, the equations behaved differently compared to the Sasaki-Einstein canonical connection of the Calabi-Yau cone of section 4. Nonetheless, the overall picture remains: the Nahm-like equations are expected to have a Kähler structure on the moduli space and the precise treatment of boundary conditions only changes the orbit into which the space is embedded to.

However, the structure of the entire intersection of the single HYM moduli spaces is not yet fully understood. The complications can be traced back to the different bundle structure of 3-Sasakian manifolds as $\mathrm{SU}(2)$ (or $\mathrm{SO}(3)$ )-bundle, while in the usual SasakiEinstein case one had a U(1)-bundle over the underlying space. The latter allowed for 


\begin{tabular}{|c|c|c|c|}
\hline & $\mathfrak{s u}(n+1)$ & $\mathfrak{s u}(n)$ & $\mathfrak{m}$ \\
\hline CSA & $H_{\alpha_{i}}, i=1, \ldots, n$ & $H_{\alpha_{i}}, i=1, \ldots, n-1$ & $H_{\alpha_{n}}$ \\
simple roots & $E_{e_{i}-e_{i+1}}, i=1, \ldots, n$ & $E_{e_{i}-e_{i+1}}, i=1, \ldots, n-1$ & $E_{e_{n}-e_{n+1}}$ \\
positive roots & $E_{e_{i}-e_{j}}, 1 \leq i<j \leq n+1$ & $E_{e_{i}-e_{j+1}}, 1 \leq i<j \leq n$ & $E_{e_{i}-e_{n+1}}, i=1, \ldots, n$ \\
\hline
\end{tabular}

Table 1. The roots are given in terms of the ONB $e_{i}$ on $\mathbb{R}^{n+1}$. See for instance [52].

a direct generalization of the Nahm-type equations on Calabi-Yau cones [21], while the complete discussion of the hyper-Kähler case may require new approaches and is left for future work. By virtue of the absolutely regular formulation of hyper-Kähler instantons, one expect a generic description for all $m$, once the case of $m=1$ is understood, similarly to the generic results obtained in [21].

\section{Acknowledgments}

We are grateful to Fabio Apruzzi, Felix Lubbe, Alexander D. Popov, and Markus Röser for valuable discussions and comments. This work was done within the framework of the DFG project LE 838/13. JG is supported by the DFG research training group GRK1463 "Analysis, Geometry, and String Theory". MS is supported by Austrian Science Fund (FWF) grant P28590.

\section{A Details on non-regular boundary conditions}

We provide the details for adaptation of Kronheimer's treatment of the Nahm's equations in [42]; in particular, focusing on ([42], Lemma 10 \& Lemma 11).

Preliminaries. For a semi-simple Lie algebra of rank $k$ with simple roots $\alpha_{i}, i=1, \ldots, r$, we recall the Chevalley basis

$$
\begin{aligned}
{\left[H_{\alpha_{i}}, H_{\alpha_{j}}\right] } & =0, \\
{\left[H_{\alpha_{i}}, E_{\alpha_{j}}\right] } & =A_{j i} E_{\alpha_{j}}, \\
{\left[E_{-\alpha_{i}}, E_{\alpha_{i}}\right] } & =H_{\alpha_{i}}, \\
\left(\operatorname{ad}_{E_{ \pm \alpha_{i}}}\right)^{1-A_{j i} E_{ \pm \alpha_{j}}} & =0 .
\end{aligned}
$$

Here, $A_{j i}$ denotes the Cartan matrix elements. The last line, the Serre relations, imply that $\left[E_{\alpha_{i}}, E_{\alpha_{j}}\right]$ is non-vanishing only if $\alpha_{i}+\alpha_{j}$ is a root. Then the split $\mathfrak{s u}(n+1)=\mathfrak{s u}(n) \oplus \mathfrak{m}$ can be expressed in terms of a Cartan subalgebra (CSA) and roots as shown in table 1. Recall that $E_{e_{i}-e_{i+1}}$ are the "creation operators" for the $\mathfrak{s u}(2)$ subalgebra spanned by $\left\{H_{\alpha_{i}}, E_{ \pm\left(e_{i}-e_{i+1}\right)}\right\}$. The important question for later is whether the "creation operators" $E_{e_{i}-e_{n+1}}, i=1, \ldots, n$ on $\mathfrak{m}$ commute with each other. From the Serre relations we observe

$$
\left[E_{e_{i}-e_{n+1}}, E_{e_{j}-e_{n+1}}\right]=0
$$

because $\left(e_{i}-e_{n+1}\right)+\left(e_{j}-e_{n+1}\right)=e_{i}+e_{j}-2 e_{n+1}$ is not a root. 
Inspired from the explicit calculations for $\mathrm{SU}(3) / \mathrm{SU}(2)$ in [38] and $\mathrm{SU}(4) / \mathrm{SU}(3)$ in [35], we can replace $H_{\alpha_{n}} \in \mathfrak{m}$ by a new element $\tilde{H}$ such that

$$
\operatorname{ad}_{\tilde{H}}\left(E_{e_{i}-e_{n+1}}\right)=(n+1) E_{e_{i}-e_{n+1}} \quad \forall i=1, \ldots, n .
$$

Additionally, one can rescale $\tilde{H}$ as

$$
\tilde{H} \mapsto H=\frac{1}{n} \tilde{H}
$$

such that

$$
\operatorname{ad}_{H}\left(E_{e_{i}-e_{n+1}}\right)=\frac{n+1}{n} E_{e_{i}-e_{n+1}} \quad \forall i=1, \ldots, n
$$

This is the same rescaling as employed in the definition of the torsion components of the canonical connection $\Gamma^{P}$ of [17]. See also [38] for an explicit example in $n=2$. From now on denote

$$
E_{j}:=E_{e_{j}-e_{n+1}}, \quad F_{j}:=E_{-\left(e_{j}-e_{n+1}\right)}
$$

and note that the $E_{j}$ are nilpotent.

We now discuss the adaptation of Kronheimer's "complex trajectories" and Lemma 10 and Lemma 11 from [42] to generic Calabi-Yau cones:

Adaptation of "complex trajectory". Let $\rho_{ \pm}: \mathfrak{s u}(n+1) \rightarrow \mathfrak{g l}(p, \mathbb{C})$ be two Lie algebra homomorphisms and denote the images of the $\mathfrak{s u}(n+1)$ generators $H$ and $E_{j}$ from above as $H^{ \pm}$and $E_{j}^{ \pm}$. Then a complex trajectory is an $(n+1)$-tuple of smooth functions $\left(Y_{n+1}, Y_{j}\right): \mathbb{R} \rightarrow \mathfrak{g l}(p, \mathbb{C})$ such that

(i) the complex equations (4.7) are satisfied,

(ii) for $t \rightarrow+\infty$

$$
2 Y_{n+1}(t) \rightarrow H^{+}, \quad Y_{j}(t) \rightarrow E_{j}^{+}, \quad \forall j=1, \ldots, n
$$

(iii) and for $t \rightarrow-\infty$

$$
2 Y_{n+1}(t) \rightarrow \operatorname{Ad}_{g}\left(H^{-}\right), \quad Y_{j}(t) \rightarrow \operatorname{Ad}_{g}\left(E_{j}^{-}\right), \quad \forall j=1, \ldots, n
$$

for some $g \in \mathrm{U}(p)$ in the compact group.

Two complex trajectories $\left(Y_{n+1}, Y_{j}\right),\left(Y_{n+1}^{\prime}, Y_{j}^{\prime}\right)$ are equivalent if there exists a map $g: \mathbb{R} \rightarrow \mathrm{GL}(p, C)$ with $g \rightarrow 1$ as $t \rightarrow \infty$, such that $\left(Y_{n+1}^{\prime}, Y_{j}^{\prime}\right)=g\left(Y_{n+1}, Y_{j}\right)$. Similarly to ([42], Lemma 9$)$, if two complex trajectories are equal outside a compact subset of $\mathbb{R}$, then they are equivalent in the above sense. 
Adaptation of Lemma 10. Let $\left(Y_{n+1}, Y_{j}\right)$ be a solution of the complex equations (4.7) satisfying the boundary conditions (A.7b). Then there exists a gauge transformation $g_{-}: \mathbb{R} \rightarrow \mathrm{GL}(p, \mathbb{C})$ with $\lim _{t \rightarrow-\infty} g_{-}=$const. such that $\left(Y_{n+1}^{\prime}, Y_{j}^{\prime}\right)=g_{-}\left(Y_{n+1}, Y_{j}\right)$ is a constant solution

$$
2 Y_{n+1}^{\prime}=H^{-}, \quad Y_{j}^{\prime}=F_{j}^{-} .
$$

To see this, consider the limit $t \rightarrow-\infty$ and without loss of generality $g \equiv 1$. By the gauge transformations and boundary conditions one can find a (framed) gauge transformation such that

$$
H_{-}=\operatorname{Ad}_{g_{0}}\left(2 Y_{n+1}\right)-\frac{\mathrm{d} g_{0}}{\mathrm{~d} t} g_{0}^{-1} .
$$

The complex equations for $Y_{j}^{\prime \prime}=Y_{j}^{g_{0}}$ then reduce to

$$
\begin{aligned}
\frac{\mathrm{d}}{\mathrm{d} t} Y_{j}^{\prime \prime}+\frac{n+1}{n} Y_{j}^{\prime \prime}+\left[H^{-}, Y_{j}^{\prime \prime}\right] & =0, \\
{\left[Y_{j}^{\prime \prime}, Y_{k}^{\prime \prime}\right] } & =0,
\end{aligned}
$$

for which the general solution is of the form

$$
Y_{j}^{\prime \prime}(t)=e^{-\frac{n+1}{n} t} \operatorname{Ad}_{e^{\left(-H^{-} t\right)}}\left(\omega_{j}\right),
$$

where the $\omega_{j}$ need to commute among each other. Note that the homomorphism $\rho_{-}$induces a decomposition

$$
\mathfrak{g l}(p, \mathbb{C})=\bigoplus_{\vec{\mu} \in \mathfrak{s u}(n), i \in \mathfrak{u}(1)} V_{\vec{\mu}, i}
$$

where $\vec{\mu}$ labels representations of $\mathrm{SU}(n)$, while $i$ is the eigenvalue of $\operatorname{ad}_{H^{-}}$corresponding to the label for the $\mathrm{U}(1)$ centraliser of $\mathrm{SU}(n)$ inside $\mathrm{SU}(n+1)$.

Due to the boundary conditions we write $\omega_{j}=F_{j}^{-}+\delta_{j}$ and therefore obtain

$$
Y_{j}^{\prime \prime}(t)=F_{j}^{-}+e^{-\frac{n+1}{n} t} \operatorname{Ad}_{\left.e^{\left(-H^{-}\right.} t\right)}\left(\delta_{j}\right) .
$$

Additionally, we need to satisfy the commutator constraint in (4.7) for which we find

$$
\left[F_{j}^{-}, \delta_{k}\right]+\left[\delta_{j}, F_{k}^{-}\right]=0, \quad\left[\delta_{j}, \delta_{k}\right]=0,
$$

using that $\left[F_{j}^{-}, F_{k}^{-}\right]=0$ by the earlier arguments. Moreover, the $\operatorname{ad}_{H^{-}-\text {eigenvalues } i \text { of } \delta_{j}}$ are restricted by demanding that the $\delta_{j}$ contribution does not interfere with the boundary condition, i.e.

$$
\lim _{t \rightarrow-\infty} e^{-\frac{n+1}{n} t} \operatorname{Ad}_{e^{\left(-H^{-} t\right)}}\left(\delta_{j}\right)=0 \quad \Leftrightarrow \quad i\left(\delta_{j}\right)<-\frac{n+1}{n} .
$$

The inequality here means that $\delta_{j}$ is an element of a subspace of the decomposition (A.12), where the $\operatorname{ad}_{H^{-}}$eigenvalue is bounded by the above expression. For the case of the original 
Nahm equations, i.e. $n=1$, this yields the bound $i(\delta)<-2$ from [42]. There is still a remaining gauge freedom by

$$
g=g_{1} \cdot \ldots \cdot g_{n}, \quad g_{j}=e^{-H^{-} t} e^{\gamma_{j}} e^{H^{-} t},
$$

which acts on all $Y_{j}^{\prime}$ the same and where the contributions $g_{j}$ are suitably chosen such that they yield the desired action on each element on the tuple. More precisely, the appearing $\gamma_{j}$ are restricted by demanding

(i) $g$ preserves $2 Y_{n+1}^{\prime \prime}=H^{-}$,

(ii) $\lim _{t \rightarrow-\infty} g=1$,

(iii) $g_{j}$ preserves $Y_{k}^{\prime \prime}=F_{k}^{-}+e^{-\frac{n+1}{n} t} \operatorname{Ad}_{e^{\left(-H^{-} t\right)}}\left(\delta_{j}\right)$ for all $k \neq j$.

The first condition (i) is satisfied by noticing

$$
g=e^{-H^{-} t}\left(\prod_{j} e^{\gamma_{j}}\right) e^{H^{-} t} .
$$

The third condition (iii) requires

$$
\gamma_{j} \in \bigcap_{k \neq j} z\left(F_{k}^{-}\right), \quad\left[\gamma_{j}, \delta_{k}\right]=0=\left[\gamma_{j}, \gamma_{k}\right], \quad j \neq k,
$$

while the second condition (ii) gives a restriction on the eigenvalues of $\gamma_{j}$

$$
\lim _{t \rightarrow-\infty} g_{j}=1 \quad \Leftrightarrow \quad i\left(\gamma_{j}\right)<0 .
$$

Applying the overall gauge transformations, we arrive at

$$
Y_{k}^{\prime \prime \prime}=F_{k}^{-}+e^{-\frac{n+1}{n} t} \operatorname{Ad}_{\left.e^{\left(-H^{-}\right.} t\right)}\left(\operatorname{Ad}_{e^{\gamma_{j}}}\left(F_{j}^{-}+\delta_{j}\right)-F_{j}^{-}\right) .
$$

Similarly to [42], the only step left to prove is that for each $\delta_{j}$ there exists a $\gamma_{j}$ such that

$$
\operatorname{Ad}_{e^{\gamma_{j}}}\left(F_{j}^{-}+\delta_{j}\right)-F_{j}^{-}=0 .
$$

We have to prove a similar statement for the adaptation of Lemma 11 and provide the details there.

Adaptation of Lemma 11. Let $\left(Y_{n+1}, Y_{j}\right)$ be a solution of the complex equations (4.7) satisfying the boundary conditions (A.7a). Then there exists a unique gauge transformation $g_{+}: \mathbb{R} \rightarrow \mathrm{GL}(p, \mathbb{C})$ with $\lim _{t \rightarrow \infty} g_{+}=1$ such that $\left(Y_{n+1}^{\prime}, Y_{j}^{\prime}\right)=g_{+}\left(Y_{n+1}, Y_{j}\right)$ satisfies

$$
2 Y_{n+1}^{\prime}=H^{+}=\mathrm{const}, \quad Y_{j}^{\prime}(t=0) \in S\left(F_{j}^{+}\right) .
$$

In other words,

$$
\left(Y_{1}^{\prime}, \ldots, Y_{n}^{\prime}\right)(t=0) \in S_{\text {diag }}
$$


as defined in (4.17). The first part of the generalisation of ([42], Lemma 11) proceeds as above, the only changes are that the general solutions look $Y_{k}^{\prime \prime}=F_{k}^{+}+e^{-\frac{n+1}{n} t} \operatorname{Ad}_{e^{\left(-H^{+} t\right)}}\left(\epsilon_{j}\right)$ and the $\epsilon_{j}$ satisfy

$$
\left[\epsilon_{j}, \epsilon_{k}\right]=0, \quad\left[F_{j}^{+}, \epsilon_{k}\right]+\left[\epsilon_{j}, F_{k}^{+}\right]=0, \quad i\left(\epsilon_{j}\right)>-\frac{n+1}{n} .
$$

Note that the $i$ eigenvalues are with respect to $\mathrm{ad}_{H^{+}}$and recall that we are now considering the opposite limit compared to Lemma 10 (and therefore have the opposite inequality).

In the next step, one applies the similar gauge transformations

$$
g=g_{1} \cdot \ldots \cdot g_{n}, \quad g_{j}=e^{-H^{+} t} e^{\gamma_{j}} e^{H^{+} t},
$$

this time subject to the conditions

$$
\gamma_{j} \in \bigcap_{k \neq j} z\left(F_{k}^{+}\right), \quad\left[\gamma_{j}, \epsilon_{k}\right]=0=\left[\gamma_{j}, \gamma_{k}\right], \quad j \neq k, \quad i\left(\gamma_{j}\right)>0 .
$$

Then one needs to prove that for each $\epsilon_{j}$ there exists a unique $\gamma_{j}$ such that

$$
\operatorname{Ad}_{e^{\gamma_{j}}}\left(F_{j}^{+}+\epsilon_{j}\right)-F_{j}^{+} \in z\left(E_{j}^{+}\right) .
$$

Again, the arguments by Kronheimer apply, but let us be more explicit. The homomorphism $\rho_{+}$induces the decomposition $\left.\mathfrak{g l}(p, \mathbb{C})\right|_{\mathfrak{s l}(n+1, \mathbb{C})}=\oplus_{\kappa} V_{\kappa}$ into $\mathfrak{s l}(n+1, \mathbb{C})$ irreps $V_{\kappa}$. These decompose further under $\operatorname{Ad}_{H^{+}}$into 1-dimensional irreps $V_{\kappa}=\oplus_{i} V_{\kappa, i}$. Since $V_{\kappa}$ is an $\mathfrak{s l}(n+1, \mathbb{C})$ irrep, the highest weight vector also has the maximal $i$ eigenvalue $\lambda_{\kappa}>0$ of all weight vectors of $V_{\kappa}$.

The linearisation of (A.27) is $\epsilon_{j}-\left[F_{j}^{+}, \gamma_{j}\right] \in z\left(E_{j}^{+}\right)$. Since $F_{j}^{+}$is a annihilation operator, it decreases all $i$ eigenvalues by a certain increment $\Delta_{j}$; in other words $\operatorname{ad}_{F_{j}^{+}}: \oplus_{i>0} V_{i} \rightarrow \oplus_{i>-\Delta_{j}} V_{i}$, where $V_{i}$ are eigenspaces with certain $i$ eigenvalue. This map is injective, because the kernel of $\operatorname{ad}_{F_{j}^{+}}$is not contained in the domain since all $i>0$. Moreover, the image lies in the complement of $z\left(E_{j}^{+}\right)$, because all $i$ eigenvalues have been lowered by $\Delta_{j}$, therefore none of the weight vectors has the maximal eigenvalue and cannot be annihilated by $E_{j}^{+}$. Consequently, for any $\epsilon_{j}$ one can find a unique $\gamma_{j}$ to match the part of $\epsilon_{j}$ in the orthogonal complement of $z\left(E_{j}^{+}\right)$; hence, the claim holds.

Both Lemmata together. Consequently, a complex trajectory is equivalent to a tuple $\left(Y_{n+1}, Y_{j}\right)$ satisfying the conditions

$$
\begin{aligned}
& Y_{n+1}(t)=\frac{1}{2} H^{-}, \quad Y_{j}(t)=F_{j}^{-}, \quad t \in(-\infty, 0], \\
& Y_{n+1}(t)=\frac{1}{2} H^{+}, \quad Y_{j}(t)=F_{j}^{+}+e^{-\frac{n+1}{n} t} \operatorname{Ad}_{e^{-H^{+} t}}\left(\epsilon_{j}\right), \quad t \in[1, \infty) .
\end{aligned}
$$

The choice of $\epsilon_{j}$ is such that $\left(F_{1}^{+}+\epsilon_{1}, \ldots, F_{n}^{+}+\epsilon_{n}\right) \in S_{\operatorname{diag}}\left(\rho_{+}\right)$. Since the solution is locally constant, it follows that there exists an $g \in \mathrm{GL}(p, \mathbb{C})$ such that

$$
\operatorname{Ad}_{g}\left(F_{1}^{-}, \ldots, F_{n}^{-}\right)=\left(F_{1}^{+}+\epsilon_{1}, \ldots, F_{n}^{+}+\epsilon_{n}\right) \in \mathcal{N}_{\text {diag }}\left(\rho_{-}\right) .
$$

Hence, the complex trajectories are classified by the intersection $\mathcal{N}_{\text {diag }}\left(\rho_{-}\right) \cap S_{\operatorname{diag}}\left(\rho_{+}\right)$. 
Open Access. This article is distributed under the terms of the Creative Commons Attribution License (CC-BY 4.0), which permits any use, distribution and reproduction in any medium, provided the original author(s) and source are credited.

\section{References}

[1] M. Gross, D. Huybrechts and D. Joyce, Calabi-Yau manifolds and related geometries, Lectures from the Summer School in Nordfjordeid, Nordfjordeid Norway (2001), Springer-Verlag, Berlin Germany (2003).

[2] N.J. Hitchin, A. Karlhede, U. Lindström and M. Roček, HyperKähler Metrics and Supersymmetry, Commun. Math. Phys. 108 (1987) 535 [InSPIRE].

[3] G.W. Gibbons and P. Rychenkova, Cones, triSasakian structures and superconformal invariance, Phys. Lett. B 443 (1998) 138 [hep-th/9809158] [INSPIRE].

[4] B. de Wit, B. Kleijn and S. Vandoren, Rigid $N=2$ superconformal hypermultiplets, hep-th/9808160 [INSPIRE].

[5] S.K. Donaldson, Self-dual connections and the topology of smooth 4-manifolds, Bull. Am. Math. Soc. 8 (1983) 81.

[6] E. Corrigan, C. Devchand, D.B. Fairlie and J. Nuyts, First Order Equations for Gauge Fields in Spaces of Dimension Greater Than Four, Nucl. Phys. B 214 (1983) 452 [inSPIRE].

[7] S.K. Donaldson, Anti self-dual Yang-Mills connections over complex algebraic surfaces and stable vector bundles, Proc. London Math. Soc. 3 (1985) 1.

[8] K. Uhlenbeck and S.-T. Yau, On the existence of Hermitian-Yang-Mills connections in stable vector bundles, Commun. Pure Appl. Math. 39 (1986) 1.

[9] M. Mamone Capria and S.M. Salamon, Yang-Mills fields on quaternionic spaces, Nonlinearity 1 (1988) 517.

[10] T. Nitta, Vector bundles over quaternionic Kähler manifolds, Tohoku Math. J. 40 (1988) 425.

[11] C. Bartocci and M. Jardim, Hyperkähler Nahm transforms, CRM Proc. Lecture Notes 38 (2004) 103 [math/0312045].

[12] D. Harland, T.A. Ivanova, O. Lechtenfeld and A.D. Popov, Yang-Mills flows on nearly Kähler manifolds and $G_{2}$-instantons, Commun. Math. Phys. 300 (2010) 185 [arXiv: 0909.2730] [INSPIRE].

[13] D. Harland and A.D. Popov, Yang-Mills fields in flux compactifications on homogeneous manifolds with SU(4)-structure, JHEP 02 (2012) 107 [arXiv: 1005. 2837] [INSPIRE].

[14] I. Bauer, T.A. Ivanova, O. Lechtenfeld and F. Lubbe, Yang-Mills instantons and dyons on homogeneous $G_{2}$-manifolds, JHEP 10 (2010) 044 [arXiv: 1006.2388] [INSPIRE].

[15] A.S. Haupt, T.A. Ivanova, O. Lechtenfeld and A.D. Popov, Chern-Simons flows on Aloff-Wallach spaces and Spin(7)-instantons, Phys. Rev. D 83 (2011) 105028 [arXiv: 1104.5231] [INSPIRE].

[16] K.-P. Gemmer, O. Lechtenfeld, C. Nolle and A.D. Popov, Yang-Mills instantons on cones and sine-cones over nearly Kähler manifolds, JHEP 09 (2011) 103 [arXiv:1108.3951] [INSPIRE].

[17] D. Harland and C. Nölle, Instantons and Killing spinors, JHEP 03 (2012) 082 [arXiv:1109.3552] [INSPIRE]. 
[18] T.A. Ivanova and A.D. Popov, Instantons on Special Holonomy Manifolds, Phys. Rev. D 85 (2012) 105012 [arXiv: 1203.2657] [InSPIRE].

[19] S. Bunk, T.A. Ivanova, O. Lechtenfeld, A.D. Popov and M. Sperling, Instantons on sine-cones over Sasakian manifolds, Phys. Rev. D 90 (2014) 065028 [arXiv:1407.2948] [INSPIRE].

[20] S. Bunk, O. Lechtenfeld, A.D. Popov and M. Sperling, Instantons on conical half-flat 6-manifolds, JHEP 01 (2015) 030 [arXiv:1409.0030] [INSPIRE].

[21] M. Sperling, Instantons on Calabi-Yau cones, Nucl. Phys. B 901 (2015) 354 [arXiv: 1505.01755] [INSPIRE].

[22] A.S. Haupt, Yang-Mills solutions and Spin(7)-instantons on cylinders over coset spaces with $G_{2}$-structure, JHEP 03 (2016) 038 [arXiv:1512.07254] [INSPIRE].

[23] D. Gaiotto and E. Witten, Supersymmetric Boundary Conditions in $N=4$ Super Yang-Mills Theory, J. Statist. Phys. 135 (2009) 789 [arXiv:0804.2902] [INSPIRE].

[24] D. Gaiotto and E. Witten, S-duality of Boundary Conditions In $N=4$ Super Yang-Mills Theory, Adv. Theor. Math. Phys. 13 (2009) 721 [arXiv:0807.3720] [InSPIRE].

[25] D. Xie, M5 brane and four dimensional $N=1$ theories I, JHEP 04 (2014) 154 [arXiv: 1307.5877] [INSPIRE].

[26] J.J. Heckman, P. Jefferson, T. Rudelius and C. Vafa, Punctures for theories of class $\mathcal{S}_{\Gamma}$, JHEP 03 (2017) 171 [arXiv:1609.01281] [INSPIRE].

[27] A. Hashimoto, P. Ouyang and M. Yamazaki, Boundaries and defects of $\mathcal{N}=4$ SYM with 4 supercharges. Part I: Boundary/junction conditions, JHEP 10 (2014) 107 [arXiv: 1404.5527] [INSPIRE].

[28] A. Hashimoto, P. Ouyang and M. Yamazaki, Boundaries and defects of $\mathcal{N}=4$ SYM with 4 supercharges. Part II: Brane constructions and $3 d \mathcal{N}=2$ field theories, JHEP 10 (2014) 108 [arXiv: 1406.5501] [INSPIRE].

[29] C.P. Boyer and K. Galicki, 3-Sasakian manifolds, Surveys Diff. Geom. 7 (1999) 123 [hep-th/9810250] [INSPIRE].

[30] C.P. Boyer, K. Galicki and B.M. Mann, The geometry and topology of 3-Sasakian manifolds, J. Reine Angew. Math. 455 (1994) 183.

[31] N. Hitchin, Hyperkähler manifolds, Séminaire Bourbaki 34 (1991-1992) 137.

[32] H. Baum, T. Friedrich, R. Grunewald and I. Kath, Teubner-Texte zur Mathematik. Vol. 124: Twistors and Killing spinors on Riemannian manifolds, B.G. Teubner, Leipzig Germany (1991).

[33] M.F. Atiyah and R. Bott, The Yang-Mills equations over Riemann surfaces, Phil. Trans. Roy. Soc. Lond. A 308 (1982) 523 [InSPIRE].

[34] A. Deser, O. Lechtenfeld and A.D. Popov, $\sigma$-model limit of Yang-Mills instantons in higher dimensions, Nucl. Phys. B 894 (2015) 361 [arXiv:1412.4258] [InSPIRE].

[35] J.C. Geipel, O. Lechtenfeld, A.D. Popov and R.J. Szabo, Sasakian quiver gauge theories and instantons on cones over round and squashed seven-spheres, arXiv:1706.07383 [INSPIRE].

[36] O. Lechtenfeld, A.D. Popov and R.J. Szabo, SU(3)-Equivariant Quiver Gauge Theories and Nonabelian Vortices, JHEP 08 (2008) 093 [arXiv: 0806.2791] [INSPIRE]. 
[37] B.P. Dolan and R.J. Szabo, Equivariant Dimensional Reduction and Quiver Gauge Theories, Gen. Rel. Grav. 43 (2010) 2453 [arXiv: 1001.2429] [INSPIRE].

[38] O. Lechtenfeld, A.D. Popov, M. Sperling and R.J. Szabo, Sasakian quiver gauge theories and instantons on cones over lens 5-spaces, Nucl. Phys. B 899 (2015) 848 [arXiv:1506. 02786] [INSPIRE].

[39] J.C. Geipel, O. Lechtenfeld, A.D. Popov and R.J. Szabo, Sasakian quiver gauge theories and instantons on the conifold, Nucl. Phys. B 907 (2016) 445 [arXiv:1601.05719] [INSPIRE].

[40] J.C. Geipel, Sasakian quiver gauge theory on the Aloff-Wallach space $X_{1,1}$, Nucl. Phys. B 916 (2017) 279 [arXiv:1605.03521] [InSPIRE].

[41] P.B. Kronheimer, A hyper-Kählerian structure on coadjoint orbits of a semisimple complex group, J. London Math. Soc. 2 (1990) 193.

[42] P.B. Kronheimer, Instantons and the geometry of the nilpotent variety, J. Diff. Geom. 32 (1990) 473 [INSPIRE].

[43] S.K. Donaldson, Nahm'S equations and the classification of monopoles, Commun. Math. Phys. 96 (1984) 387 [InSPIRE].

[44] O. Lechtenfeld, Instantons and Chern-Simons flows in 6, 7 and 8 dimensions, Phys. Part. Nucl. 43 (2012) 569 [arXiv:1201.6390] [InSPIRE].

[45] M. Sperling, Two aspects of gauge theories: higher-dimensional instantons on cones over Sasaki-Einstein spaces and Coulomb branches for 3-dimensional $N=4$ gauge theories, Ph.D. Thesis, Hannover University, Hannover Germany (2016).

[46] V. Ginzburg, Principal nilpotent pairs in a semisimple Lie algebra, Invent. Math. 140 (2000) 511 [math/9903059].

[47] D.I. Panyushev, Nilpotent pairs in semisimple Lie algebras and their characteristics, Int. Math. Res. Notices 2000 (2000) 1 [math/9906049].

[48] D.I. Panyushev, Nilpotent pairs, dual pairs, and sheets, J. Algebra 240 (2001) 635 [math/9904014].

[49] A.G. Elashvili and D.I. Panyushev, A classification of the principal nilpotent pairs in simple Lie algebras and related problems, J. London Math. Soc. 63 (2001) 299 [math/9909082].

[50] O. Biquard, Sur les équations de Nahm et la structure de Poisson des algèbres de Lie semi-simples complexes, Math. Ann. 304 (1996) 253.

[51] A.G. Kovalev, Nahm's equations and complex adjoint orbits, Quart. J. Math. 47 (1996) 41.

[52] J. Fuchs and C. Schweigert, Symmetries, Lie algebras and representations: A graduate course for physicists, Cambridge University Press, Cambridge U.K. (2003). 